\title{
Structural and biochemical mechanisms of NLRP1 inhibition by DPP9
}

https://doi.org/10.1038/s41586-021-03320-w

Received: 14 August 2020

Accepted: 1 February 2021

Published online: 17 March 2021

Open access

Check for updates

\author{
Menghang Huang ${ }^{1,8}$, Xiaoxiao Zhang ${ }^{1,8}$, Gee Ann Toh ${ }^{2}$, Qin Gong $^{3}$, Jia Wang ${ }^{1}$, Zhifu Han', $^{1}$ \\ Bin $\mathrm{Wu}^{3,4}$, Franklin Zhong ${ }^{2,5 \bowtie}$ \& Jijie Chai ${ }^{1,6,7 凶}$
}

Nucleotide-binding domain, leucine-rich repeat receptors (NLRs) mediate innate immunity by forming inflammasomes. Activation of the NLR protein NLRP1 requires autocleavage within its function-to-find domain (FIIND) ${ }^{1-7}$. In resting cells, the dipeptidyl peptidases DPP8 and DPP9 interact with the FIIND of NLRP1 and suppress spontaneous NLRP1 activation ${ }^{8,9}$; however, the mechanisms through which this occurs remain unknown. Here we present structural and biochemical evidence that full-length rat NLRP1 (rNLRP1) and rat DPP9 (rDPP9) form a 2:1 complex that contains an autoinhibited rNLRP1 molecule and an active UPA-CARD fragment of rNLRP1. The ZU5 domain is required not only for autoinhibition of rNLRP1 but also for assembly of the 2:1 complex. Formation of the complex prevents UPA-mediated higher-order oligomerization of UPA-CARD fragments and strengthens ZU5-mediated NLRP1 autoinhibition. Structure-guided biochemical and functional assays show that both NLRP1 binding and enzymatic activity are required for DPP9 to suppress NLRP1 in human cells. Together, our data reveal the mechanism of DPP9-mediated inhibition of NLRP1 and shed light on the activation of the NLRP1 inflammasome.
In the mammalian innate immune system, the detection of pathogen-derived or host-derived signals by NLRs induces their oligomerization, forming multiprotein complexes called inflammasomes that mediate inflammatory cell death and cytokine secretion ${ }^{10}$. NLRs generally consist of an $\mathrm{N}$-terminal domain, a central nucleotide-binding and oligomerization domain (NOD) and a C-terminal leucine-rich repeat (LRR) domain. NLRP1, like CARD8 ( refs. $^{4,7}$ ), contains an unusual domain known as FIIND ${ }^{11}$ (Fig. 1a), which contains the subdomains ZU5 (found in the tight-junction protein ZO-1 and the netrin receptor UNC5) and UPA (conserved in UNC5, the death-domain-containing protein PIDD and proteins of the ankyrin family). Autoproteolysis between these two subdomains is a prerequisite for the activation of NLRP1 (refs. ${ }^{4,7}$ ) and of CARD8 (ref. ${ }^{12}$ ). Bacillus anthracis lethal factor is the best-characterized pathogen-derived trigger for the activation of rodent NLRP1 (refs. ${ }^{5,13}$ ). Lethal factor cleaves mouse NLRP1B close to its N terminus and induces proteasomal degradation of the entire N-terminal NOD-LRR-ZU5 fragment via the $\mathrm{N}$-end rule pathway ${ }^{1-3}$. This liberates the active UPA-CARD fragment that rapidly oligomerizes to engage downstream inflammasome effectors such as apoptosis-associated speck-like protein containing a CARD (ASC) and pro-caspase- 1 (refs. ${ }^{1,3}$ ). This unique mechanism involving 'functional degradation' is conserved in the activation of human NLRP1 (hNLRP1) by the 3 C proteases of enteroviruse ${ }^{14}$ and in the activation of CARD 8 by HIV-1 protease ${ }^{15}$.

DPP8 and DPP9 are related intracellular prolyl peptidases that are implicated in immune regulation and in other viral cellular processes $^{16}$. They are endogenous inhibitors of the NLRP1 inflammasome in humans $s^{8,9,12}$ and in rodents ${ }^{6,8,12,17,18}$. Notably, the FIIND of hNLRP1 is necessary and sufficient for interaction with human DPP9 (hDPP9) (ref. ${ }^{9}$ ). Furthermore, inhibitors of class IV DPPs-such as valine boroproline (VbP) - or knockout of DPP8 and DPP9 specifically activate NLRP1 and/or CARD8 (refs. ${ }^{6,8,9,12}$ ). Like lethal factor, VbP also induces the proteasome-mediated degradation of the NLRP1B N-terminal fragment, but this induction is independent of $\mathrm{N}$-degron recognition. VbP and lethal factor therefore trigger rodent NLRP1 activation through two distinct, independent pathways. At present, the mechanisms that underlie the DPP9-mediated inhibition of NLRP1 remain unknown.

\section{Crystal structure of the autoinhibited FIIND}

A crystal structure of the rNLRP1 FIIND of rNLRP1 shows that the purified protein is autocleaved at the predicted position between F968 and S969 (Extended Data Table 1, Extended Data Fig. 1a). This is further confirmed by SDS-PAGE analysis of the crystals (Extended Data Fig. 1b). The structure of rNLRP1 FIIND resembles that of the autoinhibited netrin receptor UNC5b ${ }^{19}$ (Extended Data Fig. 1c). Inter-domain interaction between ZU5 and UPA largely involves the first $\beta$-strand ( $\beta 13$ ) of UPA, which forms two anti- $\beta$ sheets with ZU5 (Fig. 1b); this explains how the ZU5 domain can block the release of the active UPA-CARD fragment ${ }^{4,7}$.

The catalytically essential FS motif ${ }^{20}$ is conserved in NLRP1 homologues and in CARD8 (Extended Data Fig. 1d). In the structure of rNLRP1, F968 from this motif points into a hydrophobic pocket (Fig. 1c), as is observed for the corresponding phenylalanine residue in the FIIND-containing proteins NUP98 (ref. ${ }^{21}$ ) and PIDD ${ }^{22}$. H942 of rNLRP1, which is highly conserved in NLRP1 proteins and in CARD8

'Beijing Advanced Innovation Center for Structural Biology, Tsinghua-Peking Joint Center for Life Sciences, Center for Plant Biology, School of Life Sciences, Tsinghua University, Beijing, China. ${ }^{2}$ Lee Kong Chian School of Medicine, Nanyang Technological University, Singapore, Singapore. ${ }^{3}$ School of Biological Sciences, Nanyang Technological University, Singapore,

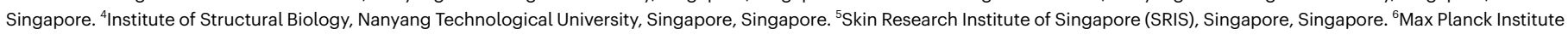

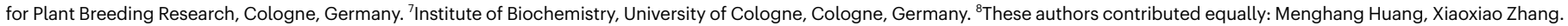
$凶$ e-mail: franklin.zhong@ntu.edu.sg; chaijj@mail.tsinghua.edu.cn 


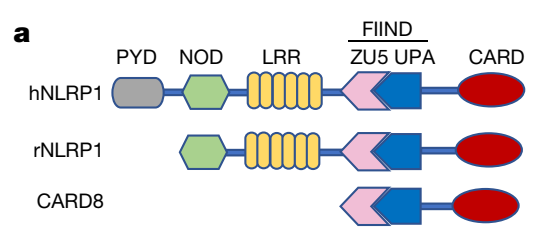

b

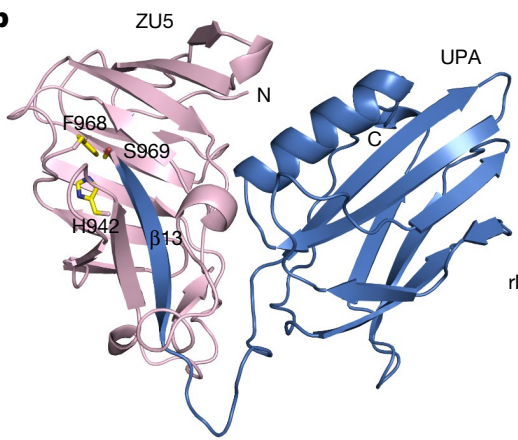

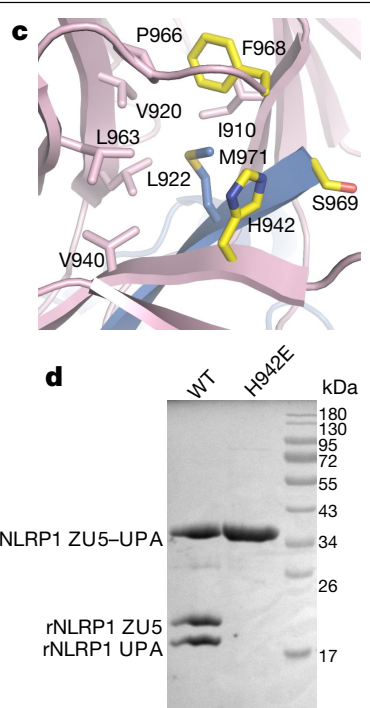

Fig. 1 Crystal structure of the FIIND of rNLRP1. a, Schematic of domain structures of hNLRP1, rNLRP1 and CARD8. CARD, caspase activation and recruitment domain; PYD, pyrin domain. b, Crystal structure of the rNLRP1 FIIND. The ZU5 and UPA subdomains are shown in pink and blue, respectively. The catalytic residues of the FIIND are labelled and shown in stick representation. c, A close-up view of the catalytic site of the FIIND. d, Mutation of the catalytic residue $\mathrm{H} 942$ abolishes autoproteolysis of the rNLRP1 FIIND. Wild-type and $\mathrm{H} 942$ mutant rNLRP1 FIIND proteins were purified from insect cells and visualized by SDS-PAGE followed by Coomassie blue staining. See Supplementary Fig. 1 for gel raw data.

(Extended Data Fig.1d), is located adjacent to 5969 from the FS motif (Fig. 1c). Mutation of H942 of rNLRP1 resulted in a complete loss of autocleavage (Fig. 1d), as was previously observed after mutation of the corresponding residues $\mathrm{H} 270$ of CARD8 or H1186 of hNLRP1 $\left(\right.$ ref. $^{20}$ ).
Collectively, these data suggest that $\mathrm{H} 942$ of rNLRP1 is a catalytic residue.

\section{Architecture of the 2:1 rNLRP1-rDPP9 complex}

Gel-filtration experiments confirmed the formation of a stable complex between full-length rNLRP1 and rDPP9 proteins purified from insect cells (Extended Data Fig. 2a)-consistent with the results of previous studies $^{3,8,9}$. A similar result was also obtained using hDPP9 and CARD8 (Extended Data Fig. 2b). After purification by gel filtration, the rNLRP1rDPP9 complex was analysed by cryo-electron microscopy (cryo-EM) (Extended Data Fig. 3). Two-dimensional class averages showed that rDPP9 formed dimers, but only one subunit was bound to NLRP1 in most of the particles (Extended Data Fig. 3b). We used the particles with one rDPP9 subunit bound by rNLRP1 for further cryo-EM analysis. After 3D classification, a subset of 182,116 particles was used for final 3D reconstruction, generating a map with a global resolution of $3.18 \AA$ (Fig. 2a, Extended Data Fig. 3c-e, Extended Data Table 2).

rDPP9 forms a homodimer (Fig. 2a) that is nearly identical to the dimeric hDPP9 $\left(\right.$ ref. $^{23}$ ). Several flexible loop regions were not observed in the reported apo-hDPP9 structure, but their equivalents are well defined in the structure of the rNLRP1-bound rDPP9 (Extended Data Fig. 4a). Unexpectedly, the liganded rDPP9 subunit is bound by two rNLRP1 molecules (Fig. 2a), which we hereafter term the 2:1 rNLRP1rDPP9 complex. The first rNLRP1 molecule contains the complete FIIND, which has a conformation nearly identical to that observed in the crystal structure of the free FIIND (Extended Data Fig. 4b); the remaining domains of this rNLRP1 molecule are not discernible in the cryo-EM density. In the second rNLRP1 molecule, only the UPA domain is well defined.

Three surfaces mediate the formation of the 2:1 rNLRP1-rDPP9 complex (Fig. 2a). The first surface is mediated by ZU5 of rNLRP1, which binds to one lateral side of the $\beta$-propeller domain of rDPP9 (termed the ZU5-binding site of rDPP9) (Fig. 2a, b, red box). The second interface is formed by the deep insertion of an $\mathrm{N}$-terminal loop

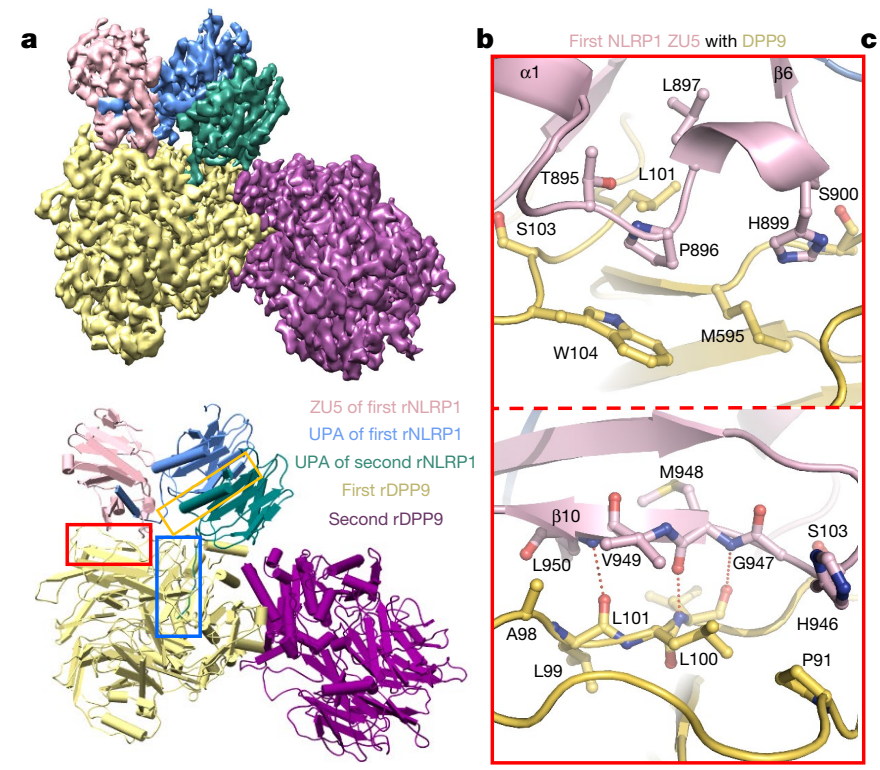

Fig. 2| Assembly mechanism of the 2:1 rNLRP1-rDPP9 complex. a, Top, the final cryo-EM density of the rNLRP1-rDPP9 complex at $3.18 \AA$. Colour codes for domain structures are indicated. Bottom, model of the 2:1 rNLRP1-rDPP9 complex. The three interfaces that mediate the rNLRP1-rDPP9 interaction are shown in coloured boxes. Red, ZU5-binding site; blue, UPA-binding site; yellow, UPA-UPA-binding site. b, Detailed interactions between ZU5 and rDPP9 at the ZU5-binding site within the red-framed region in a. Hydrogen-bonding interactions are indicated by red dashed lines. c, Detailed interactions between

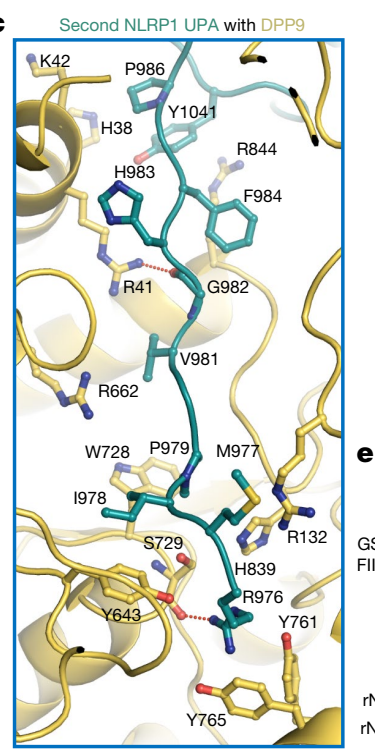

d
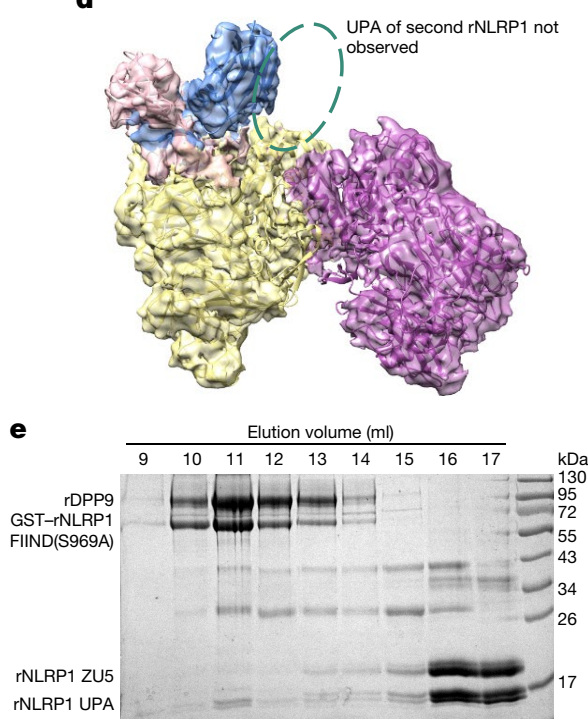

UPA and rDPP9 at the UPA-binding site within the blue-framed region in a.d, 3D reconstruction of the rNLRP1 FIIND-CARD(S969A)-rDPP9 complex.

Highlighted within the open ellipse is the vacant UPA-binding site.e, The ZU5 subdomain is displaced after interaction of the second rNLRP1 with rDPP9. The preformed rNLRP1 FIIND(S969A)-rDPP9 complex was incubated with fully autoprocessed rNLRP1 FIIND and analysed by gel-filtration experiments. Protein fractions from the gel-filtration assay were visualized by SDS-PAGE followed by Coomassie-blue staining. See Supplementary Fig. 1 for gel raw data. 
(N-loop) from the UPA domain of the second rNLRP1 molecule into the rDPP9 substrate-binding channel (termed the UPA-binding site) (Fig. 2a, c, blue box). Notably, the UPA N-loop forms a $\beta$-sheet with ZU5 in the autoinhibited FIIND (Fig. 1b). Additionally, the homodimerization interface of the two UPA domains also contributes to formation of the 2:1 complex (termed the UPA dimerization site) (Fig. 2a, yellow box).

\section{Specific interaction between rNLRP1 and rDPP9}

The interactions within the ZU5-interacting site consist of extensive contacts between $\beta 10$, the $\alpha 1-\beta 6$ loop of the rNLRP1 FIIND ZU5 subdomain, and the $\beta$-propeller domain of rDPP9 (Fig. 2b). $\beta 10$ of FIIND forms three main-chain hydrogen bonds with the $\mathrm{N}$-terminal portion of a long loop of rDPP9, forming an anti- $\beta$-sheet-like structure (Fig. $2 b$ ). The rDPP9 loop also makes hydrophobic contacts with the middle part of the $\alpha 1-\beta 6$ loop of FIIND. The amino acids of this ZU5-interacting site are conserved in DPP8 but not in DPP4 (Extended Data Fig. 4c), which explains why DPP4 fails to inhibit NLRP1B ${ }^{8,24}$. In addition, the short $\alpha$-helix in the $\alpha 1-\beta 6$ loop of FIIND packs against the other two loops of rDPP9. The substrate-binding groove of rDPP9 is completely blocked by the UPA N-loop (Fig. 2c). Consistent with previous studies $^{8,9,25}$, N-terminal sequencing indicated that the seven non-structured, $\mathrm{N}$-terminal residues of the UPA N-loop were not cleaved by DPP9 (Extended Data Fig. 5a).

\section{rNLRP1(S969A) forms a 1:1 complex with rDPP9}

The catalytically inactive mutant rDPP9(S729A)-in which the serine residue at position 729 is mutated to alanine-formed a stable complex with the rNLRP1FIIND-CARD fragment, as determined by gel-filtration experiments (Extended Data Fig. 5b). rNLRP1 FIIND in the first bound position adopts a nearly identical conformation to that of free rNLRP1 FIIND in the crystal structure (Extended Data Fig. 4b), which suggests that a non-autocleavable rNLRP1 can interact with rDPP9. Indeed, the auto-cleavage mutant fragment rNLRP1FIIND-CARD(S969A) retained rDPP9-binding activity (Extended Data Fig. 5c), consistent with previous observations ${ }^{8,12}$. Notably, the cryo-EM structure of rDPP9 in complex with rNLRP1 FIIND-CARD(S969A) (Extended Data Fig. 6) is nearly identical to that of the wild-type complex encompassing the first rNLRP1 molecule, except that no clear density was found in the second rNLRP1-binding position (Fig. 2d). This result indicates that rNLRP1 FIIND-CARD(S969A) can interact with the ZU5-binding site but not with the UPA-binding site, establishing that autoproteolysis is required for rNLRP1 to fit into the second position to form the 2:1 complex. This could also explain why autoproteolysis-deficient NLRP1 mutants retain DPP9-binding activity, but to a lesser extent ${ }^{8,9}$.

The ZU5 domain of rNLRP1 was not observed in the second position (Fig. 2a), because when rNLRP1 docks into this position this domain becomes either flexible or dissociated. To differentiate between these two possibilities, we tested whether an autocleaved rNLRP1 FIIND fits into the second position in a preformed 1:1 rNLRP1FIIND(S969A)-rDPP9 complex. Gel filtration showed that the autocleaved FIIND did interact with rNLRP1 FIIND(S969A)-rDPP9 (Fig. 2e). In the resulting complex, the ZU5 and UPA subdomains of rNLRP1 became sub-stoichiometric, which indicates that ZU5 dissociates from UPA after rNLRP1 binding in the second position.

\section{NLRP1 blocking by DPP9 enzyme and binding activity}

Point mutations at S900 of rNLRP1 and L101 of rDPP9, which were predicted to disrupt the ZU5-binding site, were found to abrogate or markedly compromise the interaction between rNLRP1 and rDPP9 (Fig. 3a, b, Extended Data Fig. 7a). This suggests that the first binding position is required in order for the second rNLRP1 to bind rDPP9.
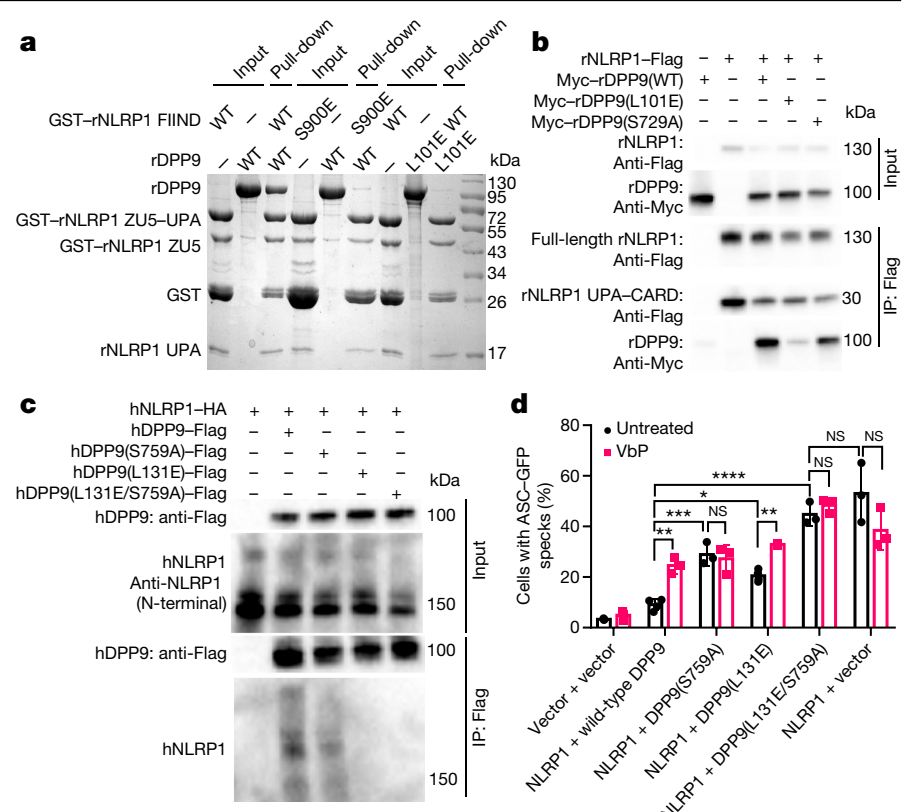

d

Anti-Myc

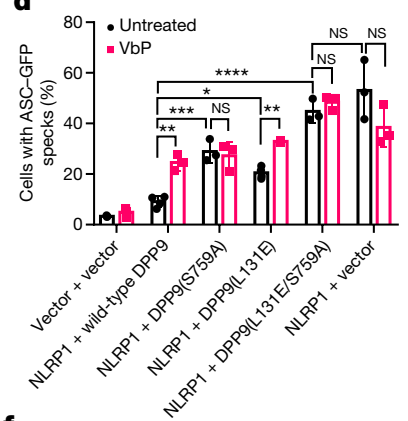

e
Doxycycline-induced
hDPP9-Flag

hDPP9-Flag

Drug treatment

Flag

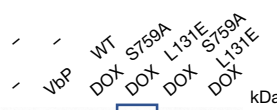

f

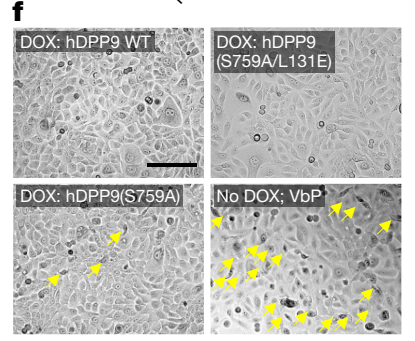

Fig. 3 Both protease activity and FIIND binding are important for the inhibition of NLRP1 by DPP9. a, rNLRP1 FIIND with an N-terminal GST tag (GST-NLRP1 FIIND) was used to pull down non-tagged rDPP9 in vitro. b, Lysates from 293T cells transfected with the indicated Flag-tagged rNLRP1 and Myc-tagged rDPP9 proteins were subjected to anti-Flag immunoprecipitation (IP). c, As in b using Flag-hDPP9 and haemagglutinin (HA)-tagged hNLRP1. d, ASC-GFP DPP8/DPP9 double-knockout 293T cells were transfected with the indicated constructs and treated with and without $\mathrm{VbP}$ for $24 \mathrm{~h}$. Bar graphs represent data from biological triplicates. Two-way ANOVA, ${ }^{*} P<0.05$, ${ }^{* *} P<0.01,{ }^{* * *} P<0.001,{ }^{* * *} P<0.0001, \mathrm{NS}$, not significant. e, Immortalized N/ TERT-1 keratinocytes stably transduced with Tet-ON-3×Flag DPP9 lentiviruses were treated with either doxycycline (DOX) or VbP for $24 \mathrm{~h}$. Lysates and the culture media were analysed by immunoblotting. f, Images of N/TERT-1 keratinocytes treated as in e. Pyroptotic cells are indicated by yellow arrows. Scale bar, $150 \mu \mathrm{m}$. See Supplementary Figs. 1 and 3 for gel raw data.

Similarly, mutation of L131 of hDPP9-the residue corresponding to L101 of rDPP9-resulted in no interaction with hNLRP1 at all in 293T cells (Fig. 3c). Notably, the corresponding mutation (L131E) in hDPP9 also resulted in a loss of interaction with CARD8 (Extended Data Fig. 7b). This suggests that the mechanism of ZU5-mediated interaction with DPP9 might be conserved among rNLRP1, hNLRP1 and CARD8.

To determine whether the binding of NLRP1 is functionally important for its inhibition by DPP9, we imaged hDPP8/hDPP9 double-knockout 293T cells that expressed ASC-GFP and co-expressed hNLRP1 and hDPP9 variants (ref. ${ }^{9}$ ). As anticipated, wild-type hDPP9 efficiently inhibited the hNLRP1-dependent formation of ASC-GFP specks (Fig. 3d, Extended Data Fig. 7c). By contrast, the binding-deficient but enzymatically active mutant hDPP9(L131E) (Extended Data Fig. 7d) and the catalytically dead but binding-active mutant hDPP9(S759A) were both defective in doing so (Fig. 3d, Extended Data Fig. 7c). These findings indicate that both catalytic activity and binding to the FIIND are important for the hDPP9-mediated inhibition of hNLRP1. hDPP9(L131E)-but not hDPP9(S759A)-retained sensitivity towards 


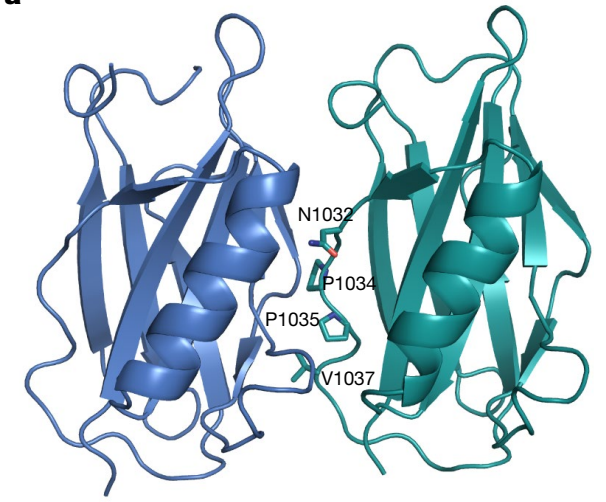

b

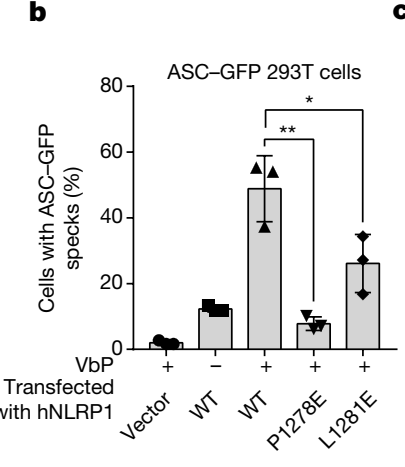

$\mathbf{f}$

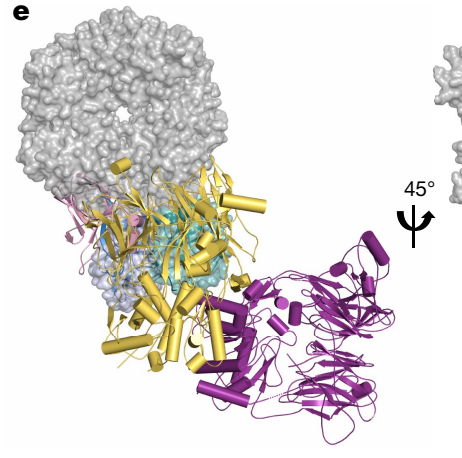

Fig. 4 | Mechanism of ZU5-mediated NLRP1 inhibition by DPP9. a, Cartoon representation of the UPA dimer in the 2:1 rNLRP1-rDPP9 complex. Key residues that mediate the formation of the dimeric UPA are shown in stick representation. b, The effect of the mutants hNLRP1(P1278E) and hNLRP1(L1281E) on the VbP-induced activation of hNLRP1 in 293T cells. Bar graphs represent data from three inductions. Student's $t$-test, ${ }^{*} P<0.05$, ${ }^{* *} P<0.01$. c, Negative staining electron microscopy analysis of human wild-type and mutant UPA-CARD fragments. Scale bar, $100 \mathrm{~nm}$. d, Alignment of the UPA homodimer from the 2:1 rNLRP1-rDPP9 complex (in cartoon) with the crystal structure of rNLRP1FIIND (shown as a transparent surface). e, Structural alignment of the oligomeric UPA-CARD fragment (shown in surface representation, PDB ID: 6K7V) with the rNLRP1-rDPP9 complex. The two UPA molecules (in blue and cyan) from the complex were used as the template for alignment.f, Working model of the DPP9-mediated inhibition of NLRP1 and the pathogen-induced activation of NLRP1. In resting cells, an
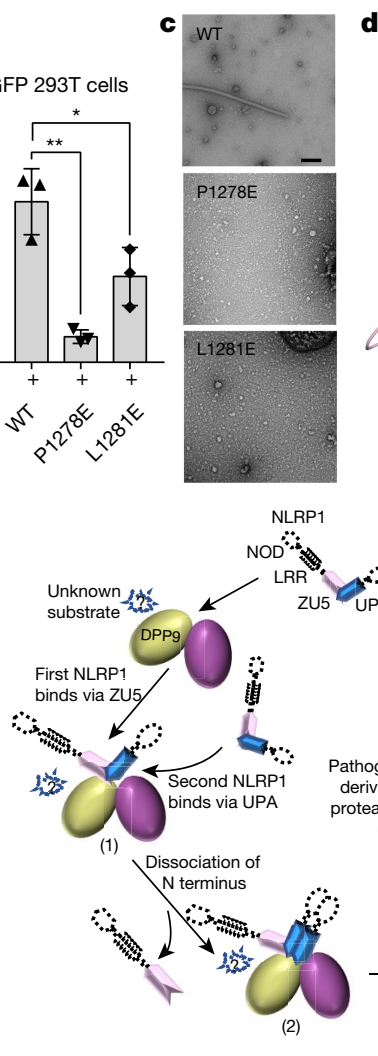

d
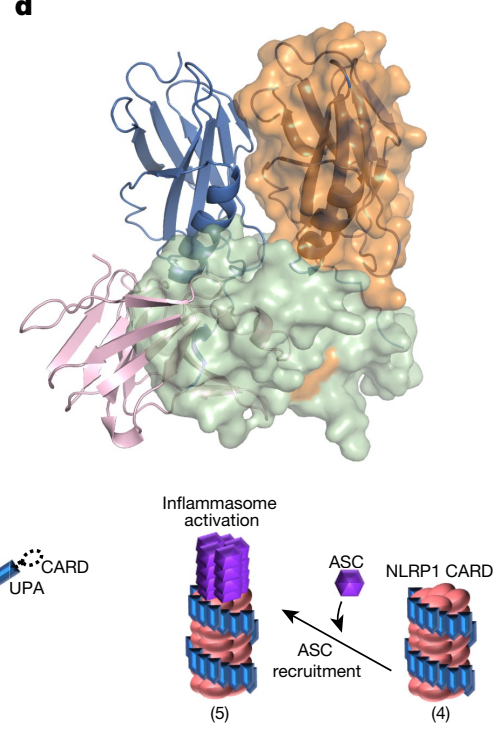

(4)

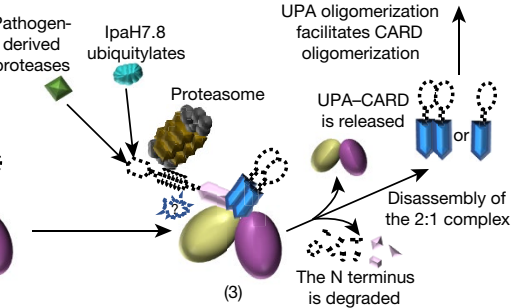

autoinhibited rNLRP1 interacts with a dimeric rDPP9 via its autoinhibitory ZU5 domain (1). This interaction enables rDPP9 to recruit an autocleaved NLRP1, resulting in dissociation of the $\mathrm{N}$-terminal segment from the $\mathrm{C}$-terminal UPACARD fragment and formation of a 2:1 rNLRP1-rDPP9 complex (2). The two UPA-CARD fragments in the complex are sequestered from oligomerization by interaction with the active sites of rDPP9 and interaction with the DPP9-bound autoinhibited rNLRP1 via UPA-UPA dimerization. There may exist a DPP9 substrate that has a role in NLRP1 inhibition, because protease activity is important in order for rDPP9 to suppress NLRP1. Such a substrate could also have a role in NLRP1 activation in the absence of DPP9. Pathogen-induced proteasomal degradation would lead to the release of the active UPA-CARD fragments from the complex (3). The released UPA-CARD fragment then oligomerizes (4) to recruit ASC for the activation of downstream immune signalling (5).

of the hNLRP1-binding-deficient hDPP9(L131E) or hDPP9(S759A/L131E) mutants-in these cells caused IL-1 $\beta$ secretion and pyroptosis (Fig. $3 e, f$, Extended Data Fig. 8a). Similar observations were also made using 293T cells expressing ASC-GFP and NLRP1 (DPP8 ${ }^{+}$DPP9 ${ }^{+}$) (Extended Data Fig. 8b). These data further support the dual requirement of the catalytic function of hDPP9 and its direct binding to hNLRP1 in order to suppress the activation of hNLRP1 in human cells.

\section{Inhibition of UPA-CARD oligomerization by ZU5}

The residues N1032, P1034, P1035 and V1037, at the centre of the UPAUPA surface (Fig. 4a), are conserved among NLRP1 proteins from different species and CARD8 (Extended Data Fig. 1d). Notably, hNLRP1-2, an hNLRP1 splice isoform that lacks this loop region, displayed impaired cell-killing activity when ectopically expressed in MCF7 cells ${ }^{27}$, supporting an essential role of this loop in hNLRP1-mediated cell death. Further supporting this conclusion, mutations of P1278 and L1281 of hNLRP1from the corresponding UPA dimerization interface-substantially reduced VbP-induced ASC speck formation in ASC-GFP-expressing 293T cells (Fig. 4b, Extended Data Fig. 8c, d). 
UPA forms a ring-like oligomer, which then brings the CARDs into close proximity for the efficient, filament-like polymerization of hNLRP1 UPA-CARD ${ }^{28}$. The UPA-UPA interaction surface observed in the 2:1 NLRP1-DPP9 complex might be similar to that in the UPA-CARD filament. Indeed, the wild-type UPA-CARD fragment of hNLRP1 has been found to form filamentous structure ${ }^{28,29}$, but the UPA-UPA interface mutants-UPA-CARD(P1278E) and UPA-CARD(L1281E)-did not (Fig. 4c), which indicates that the UPA-UPA dimer interface is required for higher-order UPA oligomerization and activation of the hNLRP1 inflammasome.

VbP had little effect on the interaction of the rNLRP1FIIND with rDPP9 (Extended Data Fig. 8e), which is consistent with data from NLRP1B and CARD 8 but contrasts with those from hNLRP1 (refs. ${ }^{8,9}$ ). It is of interest to note that the linker region between UPA and CARD in hNLRP1 is longer than that in NLRP1B and CARD8 (Extended Data Figs. 1d, 8f). Deletion analysis suggested a role of the linker region in the sensitivity of rDPP9-mediated hNLRP1 inhibition to VbP (Extended Data Fig. 8f). The mutation hNLRP1(P1214R), which is associated with autoinflammatory diseases, could perturb the interaction of hNLRP1 with hDPP9 at the UPA-binding site?

In contrast to free UPA ${ }^{28,29}$, the FIIND was monomeric in solution (Extended Data Fig. 8g), which suggests that the ZU5 domain inhibits UPA dimerization or oligomerization. Supporting this notion, structural comparison revealed that ZU5 in the monomeric FIIND sterically hinders UPA dimerization in the 2:1 complex (Fig. 4d). In its activated state, the UPA-CARD fragment forms helical filaments in which dimeric UPA spirally wraps around the inner CARDs ${ }^{28}$. Alignment of the dimeric UPA from the 2:1 rNLRP1-rDPP9 complex with that from the UPA-CARD filament ${ }^{28}$ showed that ZU5 is positioned to block the spiral growth of the dimeric UPA (Fig. 4e). Collectively, these results show that ZU5 negatively regulates NLRP1 activation by directly or indirectly inhibiting the formation of UPA-CARD filaments.

\section{Discussion}

Sequestration of the potent UPA-CARD fragment in the 2:1 rNLRP1rDPP9 complex can block the UPA-mediated formation of functional UPA-CARD filaments (Fig. 4f). This is consistent with the idea that sequestration of active domains of NLRs is a general strategy in the regulation of inflammasomes ${ }^{30}$. Our data suggested that the ZU5 domain is also important for the inhibition of DPP9-independent UPA-CARD activation (Fig. 4d, Extended Data Fig. 8g). The ZU5 domain therefore seems to be critical for the negative regulation of both DPP9-independent and DPP9-dependent NLRP1 activation. The degradation of $\mathrm{N}$-terminal fragments of NLRP1-induced by either lethal toxin or 3C proteases or by chemical inhibition of DPP9-can similarly disrupt ZU5-mediated interactions and consequently release the autoinhibited and the sequestered UPA-CARD fragments in the 2:1 NLRP1-DPP9 complex (Fig. 4f). Thus, through disruption of the ZU5-dependent interaction between NLRP1 and DPP9, N-terminal degradation of NLRP1 is the unifying mechanism of NLRP1 activation ${ }^{1-3,14}$.

It remains unknown why the protease activity of DPP9 is important for NLRP1 inhibition. A plausible explanation is the existence of a substrate(s) that is required for inhibition, but how the substrate is involved is unclear. The requirement for protease activity raises the possibility that perturbations of this activity by pathogen-derived or host-derived molecules can trigger NLRP1 activation. If this is the case, the 2:1 NLRP1-DPP9 complex could represent a bona fide receptor that senses diverse signals that induce the N-terminal degradation of NLRP1 or perturb the protease activity of DPP9.

\section{Online content}

Any methods, additional references, Nature Research reporting summaries, source data, extended data, supplementary information, acknowledgements, peer review information; details of author contributions and competing interests; and statements of data and code availability are available at https://doi.org/10.1038/s41586-021-03320-w.

1. Chui, A. J. et al. N-terminal degradation activates the NLRP1B inflammasome. Science 364, 82-85 (2019).

2. $\mathrm{Xu}, \mathrm{H}$. et al. The $\mathrm{N}$-end rule ubiquitin ligase UBR2 mediates NLRP1B inflammasome activation by anthrax lethal toxin. EMBO J. 38, e101996 (2019).

3. Sandstrom, A. et al. Functional degradation: A mechanism of NLRP1 inflammasome activation by diverse pathogen enzymes. Science 364, eaau1330 (2019)

4. Finger, J. N. et al. Autolytic proteolysis within the function to find domain (FIIND) is required for NLRP1 inflammasome activity. J. Biol. Chem. 287, 25030-25037 (2012).

5. Levinsohn, J. L. et al. Anthrax lethal factor cleavage of Nlrp1 is required for activation of the inflammasome. PLoS Pathog. 8, e1002638 (2012)

6. Okondo, M. C. et al. Inhibition of Dpp8/9 activates the Nlrp1b inflammasome. Cell Chem. Biol. 25, 262-267.e5 (2018).

7. Frew, B. C., Joag, V. R. \& Mogridge, J. Proteolytic processing of Nlrp1b is required for inflammasome activity. PLoS Pathog. 8, e1002659 (2012).

8. Griswold, A. R. et al. DPP9's enzymatic activity and not its binding to CARD8 inhibits inflammasome activation. ACS Chem. Biol. 14, 2424-2429 (2019).

9. Zhong, F. L. et al. Human DPP9 represses NLRP1 inflammasome and protects against autoinflammatory diseases via both peptidase activity and FIIND domain binding. J. Biol. Chem. 293, 18864-18878 (2018).

10. Mitchell, P. S., Sandstrom, A. \& Vance, R. E. The NLRP1 inflammasome: new mechanistic insights and unresolved mysteries. Curr. Opin. Immunol. 60, 37-45 (2019).

11. Pathan, N. et al. TUCAN, an antiapoptotic caspase-associated recruitment domain family protein overexpressed in cancer. J. Biol. Chem. 276, 32220-32229 (2001).

12. Johnson, D. C. et al. DPP8/DPP9 inhibitor-induced pyroptosis for treatment of acute myeloid leukemia. Nat. Med. 24, 1151-1156 (2018).

13. Hellmich, K. A. et al. Anthrax lethal factor cleaves mouse Nlrp1b in both toxin-sensitive and toxin-resistant macrophages. PLoS ONE 7, e49741 (2012).

14. Robinson, K. S. et al. Enteroviral 3C protease activates the human NLRP1 inflammasome in airway epithelia. Science 370, eaay2002 (2020).

15. Wang, Q. et al. CARD8 is an inflammasome sensor for HIV-1 protease activity. Science https://doi.org/10.1126/science.abe1707 (2021).

16. Zhang, H., Chen, Y., Keane, F. M. \& Gorrell, M. D. Advances in understanding the expression and function of dipeptidyl peptidase 8 and 9. Mol. Cancer Res. 11, 1487-1496 (2013).

17. de Vasconcelos, N. M. et al. DPP8/DPP9 inhibition elicits canonical Nlrp1b inflammasome hallmarks in murine macrophages. Life Sci. Alliance 2, e201900313 (2019).

18. Gai, K. et al. DPP8/9 inhibitors are universal activators of functional NLRP1 alleles. Cell Death Dis. 10, 587 (2019).

19. Wang, R. et al. Autoinhibition of UNC5b revealed by the cytoplasmic domain structure of the receptor. Mol. Cell 33, 692-703 (2009).

20. D'Osualdo, A. et al. CARD8 and NLRP1 undergo autoproteolytic processing through a ZU5-like domain. PLoS ONE 6, e27396 (2011).

21. Hodel, A. E. et al. The three-dimensional structure of the autoproteolytic, nuclear pore-targeting domain of the human nucleoporin Nup98. Mol. Cell 10, 347-358 (2002).

22. Tinel, A. et al. Autoproteolysis of PIDD marks the bifurcation between pro-death caspase-2 and pro-survival NF-KB pathway. EMBO J. 26, 197-208 (2007).

23. Ross, B. et al. Structures and mechanism of dipeptidyl peptidases 8 and 9, important players in cellular homeostasis and cancer. Proc. Natl Acad. Sci. USA 115, E1437-E1445 (2018).

24. Okondo, M. C. et al. DPP8 and DPP9 inhibition induces pro-caspase-1-dependent monocyte and macrophage pyroptosis. Nat. Chem. Biol. 13, 46-53 (2017).

25. Griswold, A. R. et al. A chemical strategy for protease substrate profiling. Cell Chem. Biol. 26, 901-907.e6 (2019).

26. Zhong, F. L. et al. Germline NLRP1 mutations cause skin inflammatory and cancer susceptibility syndromes via inflammasome activation. Cell 167, 187-202.e17 (2016).

27. Hlaing, T. et al. Molecular cloning and characterization of DEFCAP-L and -S, two isoforms of a novel member of the mammalian Ced-4 family of apoptosis proteins. J. Biol. Chem. 276, 9230-9238 (2001).

28. Qin, G. et al. Structural basis for distinct inflammasome complex assembly by human NLRP1 and CARD8. Nat. Commun. 12, 188 (2021).

29. Hollingsworth, L. R. et al. Mechanism of filament formation in UPA-promoted CARD8 and NLRP1 inflammasomes. Nat. Commun. 12, 189 (2021).

30. Pedraza-Alva, G., Pérez-Martínez, L., Valdez-Hernández, L., Meza-Sosa, K. F. \& Ando-Kuri, $M$. Negative regulation of the inflammasome: keeping inflammation under control. Immunol. Rev. 265, 231-257 (2015).

Publisher's note Springer Nature remains neutral with regard to jurisdictional claims in published maps and institutional affiliations.

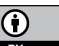

Open Access This article is licensed under a Creative Commons Attribution 4.0 International License, which permits use, sharing, adaptation, distribution and reproduction in any medium or format, as long as you give appropriate credit to the original author(s) and the source, provide a link to the Creative Commons license, and indicate if changes were made. The images or other third party material in this article are included in the article's Creative Commons license, unless indicated otherwise in a credit line to the material. If material is not included in the article's Creative Commons license and your intended use is not permitted by statutory regulation or exceeds the permitted use, you will need to obtain permission directly from the copyright holder. To view a copy of this license, visit http://creativecommons.org/licenses/by/4.0/.

(c) The Author(s) 2021 


\section{Methods}

\section{Data reporting}

No statistical methods were used to predetermine sample size. The experiments were not randomized and the investigators were not blinded to allocation during experiments and outcome assessment.

\section{Protein expression and purification}

The genes encoding full-length rNLRP1(GenBank ID: HM060632.1) and full-length rDPP9 (NCBI Reference Sequence: NM_001305241.1) were synthesized by Genewiz. The constructs of rNLRP1 (residues 1-1218), rDPP9 (residues 1-862, wild-type and all mutants), rNLRP1 FIIND (residues 822-1122, wild-type and all mutants) and rNLRP1 FIIND-CARD (residues 822-1218) were generated by a standard PCR-based cloning strategy and cloned into $\mathrm{pFastBac}-1$ vector with an $\mathrm{N}$-terminal GST tag or with no tag, and their identities were confirmed by sequencing. All the proteins were expressed using the Bac-to-Bac baculovirus expression system (Invitrogen) in sf 21 cells at $28^{\circ} \mathrm{C}$. One litre of cells $\left(2.5 \times 10^{6}\right.$ cells per $\mathrm{ml}$, medium from Expression Systems $)$ was infected with $20 \mathrm{ml}$ baculovirus at $28^{\circ} \mathrm{C}$. After growth at $28^{\circ} \mathrm{C}$ for $48 \mathrm{~h}$, the cells were collected, resuspended in the buffer containing $25 \mathrm{mM}$ Tris- $\mathrm{HCl}$ $\mathrm{pH} 8.0$ and $150 \mathrm{mM} \mathrm{NaCl}$, and lysed by sonication. The soluble fraction was purified from the cell lysate using Glutathione Sepharose 4B beads (GS4B, GE Healthcare). The proteins were then digested with PreScission protease (GE Healthcare) to remove the GST tag and further purified by gel filtration (SuperoseTM 6 prep grade XK 16/70; GE Healthcare). To prepare the rNLRP1 FIIND for crystallization trials, the purified rNLRP1 FIIND (residues 822-1122) was concentrated to about $8.0 \mathrm{mg} \mathrm{ml}^{-1}$ in buffer containing $100 \mathrm{mM} \mathrm{NaCl}, 10 \mathrm{mM}$ Tris- $\mathrm{HCl}$ $\mathrm{pH}$ 8.0. For co-expression of rNLRP1 and rDPP9, one litre of sf 21 cells were co-infected with $10 \mathrm{ml}$ recombinant baculovirus of rNLRP1 and rDPP9, and then the rNLRP1-rDPP9 complex was purified using GS4B beads. Similar protocols were used to purify the complex containing full-length GST-CARD8 and hDPP9. For cryo-EM investigation, the purified rNLRP1-rDPP9 complex was concentrated to about $0.3 \mathrm{mg} \mathrm{ml}^{-1}$ in buffer containing $25 \mathrm{mM}$ Tris- $\mathrm{HCl} \mathrm{pH} \mathrm{8.0,150} \mathrm{mM} \mathrm{NaCl} \mathrm{and} 3 \mathrm{mMDTT}$.

Recombinant hNLRP1 UPA-CARD tagged with a removable Snap domain was expressed using bacterial vectors as the form of inclusion bodies. After cellular lysis, the cellular pellet was collected after centrifugation at $30,000 \mathrm{~g}$ for $30 \mathrm{~min}$ at $4{ }^{\circ} \mathrm{C}$. Several additional washes using wash buffer ( $20 \mathrm{mM}$ Tris- $\mathrm{HCl}, \mathrm{pH} 8.0,150 \mathrm{mM} \mathrm{NaCl}, 1 \%$ Triton- $X$ and $1 \mathrm{mMDTT}$ ) were performed until a pure white pellet was obtained. The pellet was dissolved in $6 \mathrm{M}$ guanidinium, and centrifuged at $30,000 \mathrm{~g}$ for a second time for $30 \mathrm{~min}$ at room temperature to remove contaminants. The denatured soluble proteins were then gradually dialysed against 3, 2, 1.5, 1, 0.8 and 0.6 M guanidinium in dialysis buffer $(20 \mathrm{mM}$ Tris- $\mathrm{HCl}$, pH 8.0, $150 \mathrm{mM} \mathrm{NaCl}, 5 \mathrm{mM} \beta$-mercaptoethanol) in the cold room, and eventually in fresh dialysis buffer without guanidinium. The refolded proteins were centrifuged for a third time at $10,000 \mathrm{~g}$ for $10 \mathrm{~min}$ to remove misfolded aggregates. The soluble refolded fractions were then subjected to biochemical analysis and negative-stain electron microscopy experiments. The Snap tag was removed by $3 \mathrm{C}$ proteases, and the product was further purified by reverse Ni-NTA purification.

\section{Gel-filtration assay}

The GST-rNLRP1 FIIND(S969A)-rDPP9 complex and rNLRP1 FIIND proteins purified as described in the previous section were subjected to gel filtration (Superose 6,10/30; GE Healthcare) in buffer containing $10 \mathrm{mM}$ Tris pH 8.0 and $100 \mathrm{mM} \mathrm{NaCl}$. The purified rNLRP1 FIIND was left at $18^{\circ} \mathrm{C}$ for two weeks to obtain its fully autoprocessed form. The fully processed rNLRP1 FIIND was then incubated with the purified GST-rNLRP1 FIIND(S969A)-rDPP9 complex at a molar ratio of about 1:1 in $4{ }^{\circ} \mathrm{C}$ for $150 \mathrm{~min}$ before gel-filtration analysis. Samples from relevant fractions were applied to an SDS-PAGE gel and visualized by Coomassie blue staining. A similar procedure was used to assay the interaction of rDPP9 with other rNLRP1 mutant proteins.

\section{Pull-down assay}

Sf 21 cells $\left(50 \mathrm{ml} ; 2.5 \times 10^{6}\right.$ cells per $\mathrm{ml}$, medium from Expression Systems) were infected with $1 \mathrm{ml}$ baculovirus of GST-rNLRP1 FIIND (wild-type or mutants), and the proteins were expressed and purified as described in the section 'Protein expression and purification'. In brief, the proteins were purified from the cell lysate using $300 \mu \mathrm{GS} 4 \mathrm{~B}$ resin (GS4B, GE Healthcare), and incubated with an excess of purified wild-type or mutant rDPP9 proteins on ice for $60 \mathrm{~min}$. The resin was washed with $1 \mathrm{ml}$ buffer containing $10 \mathrm{mM}$ Tris pH 8.0,100 $\mathrm{mM} \mathrm{NaCl}$ five times, and eluted with $300 \mu$ l buffer containing $25 \mathrm{mM}$ Tris $\mathrm{pH}$ 8.0, $150 \mathrm{mM} \mathrm{NaCl}, 15 \mathrm{mM} \mathrm{GSH}$. The eluted samples were analysed by SDS-PAGE and visualized by Coomassie blue staining. A similar procedure was used to assay the interaction between full-length CARD8 and hDPP9.

To test the effect of VbP on the rNLRP1-rDPP9 interaction, $2 \mathrm{mM} \mathrm{VbP}$ was added to the purified rDPP9, GST-rNLRP1(S969A)-rDPP9 complex or GST-rNLRP1 FIIND-rDPP9 complex. After 60-min incubation, the samples were individually incubated with wild-type or mutant rNLRP1 FIIND and $100 \mu \mathrm{lGS} 4 \mathrm{~B}$ resin on ice for $60 \mathrm{~min}$. After extensive washing, the proteins bound in the resin were eluted and analysed by SDS-PAGE and visualized by Coomassie blue staining.

\section{Enzymatic activity assay}

To measure rDPP9 protease activity, a stock solution of substrate (10 mM Gly-Pro-AMC) was prepared in DMSO. Purified wild-type or mutant rDPP9 was diluted to $1 \mu \mathrm{M}$ to a final volume of $100 \mu \mathrm{l}$ in buffer containing $10 \mathrm{mM}$ Tris $\mathrm{pH} 8.0$ and $100 \mathrm{mM} \mathrm{NaCl}$. The substrate Gly-Pro-AMC ( $10 \mu \mathrm{l}$ of $10 \mu \mathrm{M}$ solution, dissolved in DMSO) was added to the mixture. Substrate cleavage was measured by the liberated AMC fluorescence signal recorded at room temperature in a luminescence spectrometer at excitation and emission wavelengths of $380 \mathrm{~nm}$ and $500 \mathrm{~nm}$, respectively, over a period of $30 \mathrm{~min}$.

To measure the protease activity of hDPP9, 293T cells were transfected with hDPP 9 and lysed $48 \mathrm{~h}$ post transfection in $1 \times$ Tris-buffered saline (TBS) with $0.25 \%$ NP40. Lysate $(0.3 \mu \mathrm{g})$ was mixed with $0.1 \mu \mathrm{l}$ $100 \mathrm{mM}$ Gly-Pro-AMC in $50 \mu \mathrm{l}$ lysis buffer. AMC fluorescence $(380 \mathrm{~nm}$ excitation; $500 \mathrm{~nm}$ emission) was monitored at room temperature for $30 \mathrm{~min}$ at $1-\mathrm{min}$ intervals.

\section{Edman degradation by the PPSQ-33A system}

The phenylthiohydantoin amino acid was separated in the reversed-phase mode of high-performance liquid chromatography using the differences between the retention times of different amino acids, and the amount of UV absorbance at specific wavelengths was detected. The samples were transferred to the PVDF membrane and five cycles were set. The amino acid sequences of each sample were determined from the chromatograms obtained in each cycle evaluation performed by comparing chromatograms with those in the previous and subsequent cycles and identifying the phenylthiohydantoin amino acids that had the greatest increase in abundance.

\section{Cryo-EM sample preparation and data collection}

An aliquot of $3 \mu \mathrm{l}$ of purified rNLRP1-rDPP9 or rNLRP1 FIINDCARD(S969A)-rDPP9 complex was applied to holey carbon grids (Quantifoil Au 1.2/1.3, 300 mesh), which were glow-discharged for 30 s at middle level in Harrick Plasma after 2 min evacuation. The grids were then blotted by filter papers (Ted Pella) for $2.5 \mathrm{~s}$ at $8^{\circ} \mathrm{C}$ and $100 \%$ humidity, then flash-frozen in liquid ethane using FEI Vitrobot Marke IV.

Cryo-EM data for rNLRP1-rDPP9 and rNLRP1 FIIND-CARD(S969A)rDPP9 were collected on a Titan Krios electron microscope operated at $300 \mathrm{kV}$, equipped with a Gatan K2 Summit direct electron detector and a Gatan Quantum energy filter (an additional Cs-corrector that 
was used for rNLRP1 FIIND-CARD(S969A)-rDPP9 data collection). A total of 7,157 and 4,971 micrograph stacks were automatically recorded using AutoEMation in super-resolution mode for rNLRP1-rDPP9 and rNLRP1 FIIND-CARD(S969A)-rDPP9, at a nominal magnification of $130,000 \times$ and $105,000 \times$, respectively. Defocus values varied from $1.0 \mu \mathrm{m}$ to $-2.0 \mu \mathrm{m}$ for both datasets ${ }^{31}$. Dose rates during the collection of data for rNLRP1-rDPP9 and rNLRP1 FIIND-CARD(S969A)-rDPP9 were 10 and 11 electrons per pixel per second, respectively. For both datasets, the exposure time of $5.6 \mathrm{~s}$ was dose-fractionated into $32 \mathrm{sub}$-frames, leading to a total accumulated dose of approximate 50 electrons per $\AA^{2}$ for each stack.

\section{Image processing and $3 \mathrm{D}$ reconstruction}

The stacks of rNLRP1-rDPP9 and rNLRP1 FIIND-CARD(S969A)rDPP9 recorded in super-resolution mode were motion-corrected using MotionCor 2 and binned twofold, resulting in a physical pixel size of $1.061 \AA$ per pixel and $1.091 \AA$ per pixel, respectively ${ }^{32}$. Meanwhile, dose weighting for the summed micrographs was performed ${ }^{33}$. CTFFIND4 was then used to estimate the contrast transfer function (CTF) parameters ${ }^{34}$. On the basis of the CTF estimation, 7,033 and 4,667 micrographs were manually selected for rNLRP1-rDPP9 and rNLRP1 FIIND-CARD(S969A)-rDPP9, respectively, and were further processed in Relion 3.1. Approximately 2,000 particles were manually picked and 2D-classified to generate initial templates for autopicking. In the end, 2,700,586 and 1,725,380 particles were automatically picked for rNLRP1-rDPP9 and rNLRP1FIIND-CARD(S969A)-rDPP9, respectively, using Relion 3.1. After several rounds of reference-free 2D classification, 1,430,734 particles for rNLRP1-rDPP9 and 1,117,656 particles for rNLRP1 FIIND-CARD(S969A)-rDPP9 were subjected to 3D classification, using the initial 3D reference models obtained by ab initio calculation from Relion 3.1. Particles from good 3D classes, with better overall structure features, were selected for 3D refinement. After global3D refinement and post-processing, the resolution was $3.07 \AA$ with a particle number of 343,648 for rNLRP1-rDPP9, and 3.69 $\AA$ with a particle number of 252,425 for rNLRP1 FIIND-CARD(S969A)-rDPP9.

To improve the quality of the density of the NLRP1 section in the rNLRP1-rDPP9 map, the rNLRP1-rDPP9 particles after 3D refinement were then subjected to a further round of focused 3D classification with a local mask generated using Chimera. A previously reported focused 3D classification procedure was adopted to select the 3D class with good density ${ }^{35}$. Ultimately, a subset of 182,116 particles after focused 3D classification were subjected to a final 3D refinement and yielded a global reconstruction at $3.18 \AA$ after postprocess.

$2 \mathrm{D}$ classification, $3 \mathrm{D}$ classification and $3 \mathrm{D}$ autorefinement were all performed using Relion 3.1 (refs. ${ }^{36-38}$ ). The resolutions were determined by gold-standard Fourier shell correlation ${ }^{39}$. Local resolution distribution was evaluated ${ }^{40}$ using Relion 3.1.

\section{Crystallization, data collection and structure determination}

Crystallization of rNLRP1 was performed by hanging-drop vapour-diffusion methods, mixing $1 \mu \mathrm{l}$ of $8 \mathrm{mg} \mathrm{ml}^{-1}$ protein with $1 \mu \mathrm{l}$ of reservoir solution at $18^{\circ} \mathrm{C}$. Good-quality crystals of rNLRP1 FIIND were obtained in buffer containing $1.0 \mathrm{M}$ ammonium sulfate, $0.1 \mathrm{M}$ Bis-Tris pH 5.5, 1\% w/v polyethylene glycol 3,350. All the crystals were flash-frozen in reservoir buffer to which glycerol (15\%) was added as the cryo-protectant to prevent radiation damage. The diffraction dataset was collected at the Shanghai Synchrotron Radiation Facility (SSRF) on the beamline BL19U1 using a CCD detector and was processed using HKL2000 software package. The crystal structure of rNLRP1 FIIND was determined by PHASER_MR with the structure of NUC5b as the search model. The model from the molecular replacement was manually rebuilt to the sequence of rNLRP1FIIND in the program $\operatorname{Coot}^{41}$ and subsequently subjected to refinement by the program Refine_Phenix ${ }^{42}$. Data collection, processing, and refinement statistics are summarized in Extended Data Table 1.

\section{Model building and refinement}

The EM density map of rNLRP1-rDPP9 was used for model building, as the quality of density for rNLRP1 was sufficient for sequence assignment. The model of hDPP9 (PDB ID: 6EOQ) ${ }^{23}$, along with two copies of the rNLRP1 FIIND crystal structure that we determined as described in the previous section, were docked into the EM density map of rNLRP1rDPP9 in Chimera ${ }^{43}$. The sequence of hDPP9 was changed to that of rDPP9, the whole model containing two rNLRP1 FIIND molecules and a rDPP9 dimer was then adjusted manually in the program $\operatorname{Coot}^{41}$, and refined against the EM map by Phenix in real space with secondary structure and geometry restraints ${ }^{42}$. The final model of the rNLRP1rDPP9 complex was validated using MolProbity and EMRinger in the Phenix package ${ }^{42}$. The model statistics are summarized in Extended Data Table 2.

\section{ASC-GFP transfection in 293T cells and ASC-GFP speck formation assay}

293T ASC-GFP and 293T ASC-GFP DPP8/DPP9 double-knockout cells have been previously described ${ }^{9}$. All transfections were carried out using Lipofectamine 2000 (Thermo Fisher). For immunoprecipitation, cells were collected $48 \mathrm{~h}$ post transfection. For the ASC-GFP speck assay, cells were fixed $24 \mathrm{~h}$ post transfection and counterstained with DAPI or Hoescht before wide-field fluorescence imaging. The number of nuclei per field of view was counted in ImageJ using the following image processing steps: 'Threshold' (20-30 to 255); 'Watershed'; and 'Analyze Particles' (200-infinity). ASC specks were counted in ImageJ in the GFP channel using 'Find Maxima' (prominence $=20$ ).

Inflammasome activation assays in immortalized keratinocytes Immortalized human keratinocytes (N/TERT-1) were a gift from H. Reinwald (Harvard University) (Material Transfer Agreement to Skin Research Institute of Singapore). Stably transduced N/TERT-1 cells were induced with doxycycline $\left(1 \mathrm{\mu g} \mathrm{ml}^{-1}\right)$ for $24 \mathrm{~h}$. Immunoblotting antibodies used were as follows: anti-IL-1 $\beta$ p17 specific (CST, 83186S); GAPDH (Santa Cruz Biotechnology, sc-47724); IL-1 $\beta$ (R\&D systems, AF-201); anti-Flag tag (Sigma Aldrich, F3165). All horseradish peroxidase (HRP)-conjugated secondary antibodies were purchased from Jackson Immunoresearch (goat anti-mouse IgG, 115-035-166; goat anti-rabbit IgG, 111-035-144; and donkey anti-goat IgG, 705-005-147).

\section{Reporting summary}

Further information on research design is available in the Nature Research Reporting Summary linked to this paper.

\section{Data availability}

The atomic coordinates and structure factors have been deposited in the RCSB Protein Data Bank (PDB) and Electron Microscopy Data Bank (EMDB). The PDB codes of the rNLRP1 FIIND and rNLRP1-rDPP9 structures are 7CRV and 7CRW, respectively. The EMDB codes of the rNLRP1-rDPP9 and rNLRP1 FIIND-CARD(S969A)-rDPP9 structures are EMD-30458 and EMD-30459, respectively. For gel source images, see Supplementary Information. All other data or materials can be obtained from the corresponding author upon request. Source data are provided with this paper.

\footnotetext{
31. Lei, J. \& Frank, J. Automated acquisition of cryo-electron micrographs for single particle reconstruction on an FEI Tecnai electron microscope. J. Struct. Biol. 150, 69-80 (2005).

32. Zheng, S. Q. et al. MotionCor2: anisotropic correction of beam-induced motion for improved cryo-electron microscopy. Nat. Methods 14, 331-332 (2017).

33. Grant, T. \& Grigorieff, N. Measuring the optimal exposure for single particle cryo-EM using a 2.6 Å reconstruction of rotavirus VP6. eLife 4, e06980 (2015)

34. Mindell, J. A. \& Grigorieff, N. Accurate determination of local defocus and specimen tilt in electron microscopy. J. Struct. Biol. 142, 334-347 (2003).

35. Bai, X. C., Rajendra, E., Yang, G., Shi, Y. \& Scheres, S. H. Sampling the conformational space of the catalytic subunit of human $\mathrm{y}$-secretase. eLife 4, e11182 (2015).
} 


\section{Article}

36. Scheres, S. H. A Bayesian view on cryo-EM structure determination. J. Mol. Biol. 415 406-418 (2012)

37. Scheres, S. H. RELION: implementation of a Bayesian approach to cryo-EM structure determination. J. Struct. Biol. 180, 519-530 (2012).

38. Zivanov, J., Nakane, T. \& Scheres, S. H. W. Estimation of high-order aberrations and anisotropic magnification from cryo-EM datasets in RELION-3.1. IUCrJ 7, 253-267 (2O20)

39. Rosenthal, P. B. \& Henderson, R. Optimal determination of particle orientation, absolute hand, and contrast loss in single-particle electron cryomicroscopy. J. Mol. Biol. 333 721-745 (2003).

40. Kucukelbir, A., Sigworth, F. J. \& Tagare, H. D. Quantifying the local resolution of cryo-EM density maps. Nat. Methods 11, 63-65 (2014).

41. Emsley, P., Lohkamp, B., Scott, W. G. \& Cowtan, K. Features and development of Coot. Acta Crystallogr. D 66, 486-501 (2010).

42. Adams, P. D. et al. PHENIX: a comprehensive Python-based system for macromolecular structure solution. Acta Crystallogr. D 66, 213-221 (2010).

43. Pettersen, E. F. et al. UCSF Chimera-a visualization system for exploratory research and analysis. J. Comput. Chem. 25, 1605-1612 (2004)

Acknowledgements We thank J. Lei and X. Li at Tsinghua University for data collection, and P. Schulze-Lefert at Max Planck Institute for discussion. We acknowledge support with cryo-EM facilities from the Tsinghua University Branch of the China National Center for Protein Sciences (Beijing), and support with computational facilities from the Bio-Computing Platform cluster. We thank the Shanghai Synchrotron Radiation Facility (SSRF) for assistance with data collection, and M. Han in Technology Center for Protein Science Tsinghua University for Edman sequencing analysis. This research was funded by the National Natural Science Foundation of China (31421001 to J.C.), the Alexander von Humboldt Foundation (a Humbold professorship to J.C.), Max Planck-Gesellschaft (a Max Planck fellowship to J.C.), the Ministry of Health, Singapore, NMRC grant (MOH-000382-00 to W.B.), the Concern Foundation (F.L.Z), a Nanyang Assistant Professorship (F.L.Z.) and the National Research Foundation fellowship (NRF-NRFF11-2019-0006 to F.L.Z.). We also acknowledge help and advice from members of the Reversade laboratory at Agency for Science, Technology and Research (A*STAR).

Author contributions J.C., F.Z., M.H. and Z.H. designed the project. J.C. and F.Z. supervised the project. M.H., X.Z., G.A.T., Q.G., J.W. and Z.H. performed the experiments. The data were analysed by all authors. J.C., F.Z. and B.W. wrote the manuscript with input from all authors.

Funding Open access funding provided by Max Planck Society.

Competing interests The authors declare no competing interests.

Additional information

Supplementary information The online version contains supplementary material available at https://doi.org/10.1038/s41586-021-03320-w.

Correspondence and requests for materials should be addressed to F.Z. or J.C.

Peer review information Nature thanks Zachary Billman, Edward Miao and the other, anonymous, reviewer(s) for their contribution to the peer review of this work. Reprints and permissions information is available at http://www.nature.com/reprints. 
a

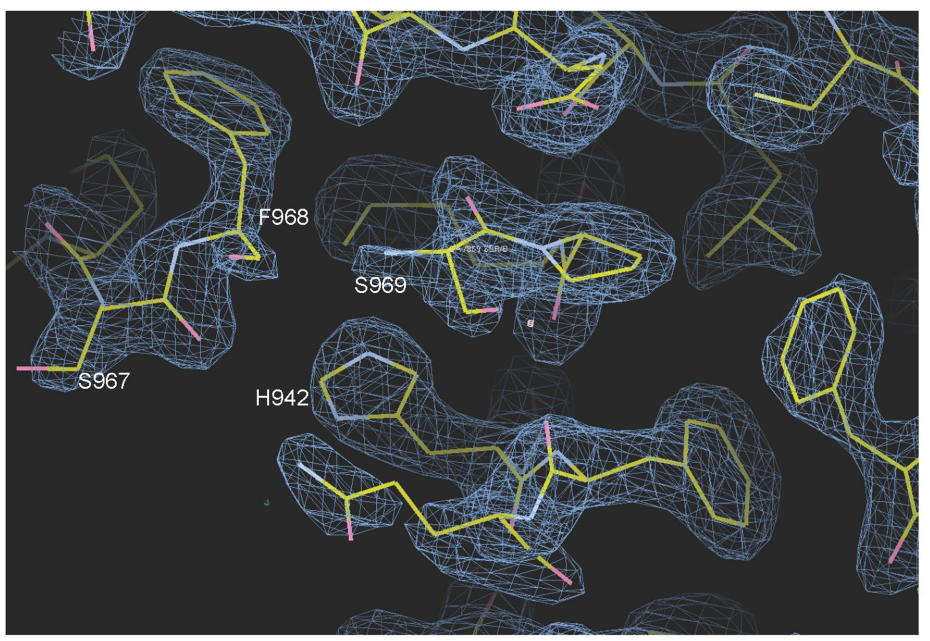

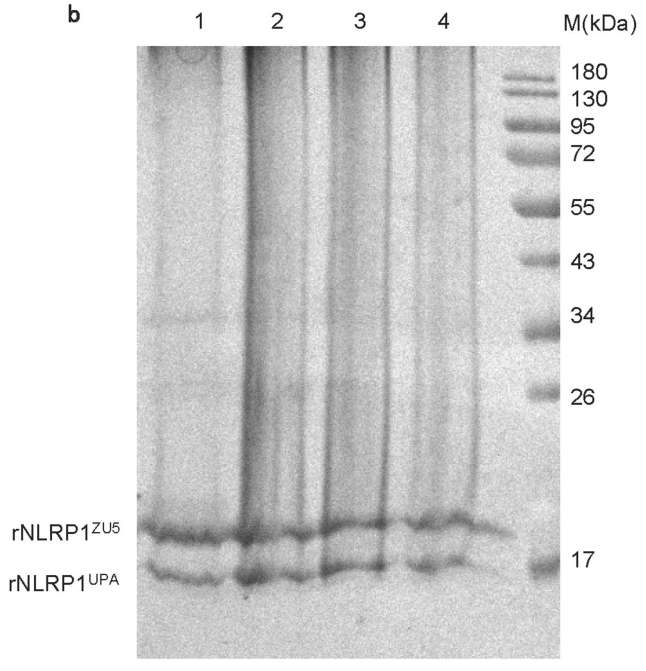

c

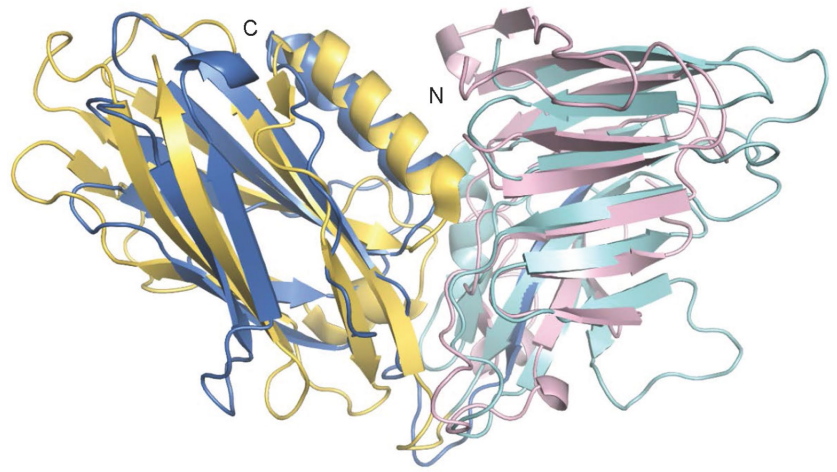

ZU5 of rNLRP

d
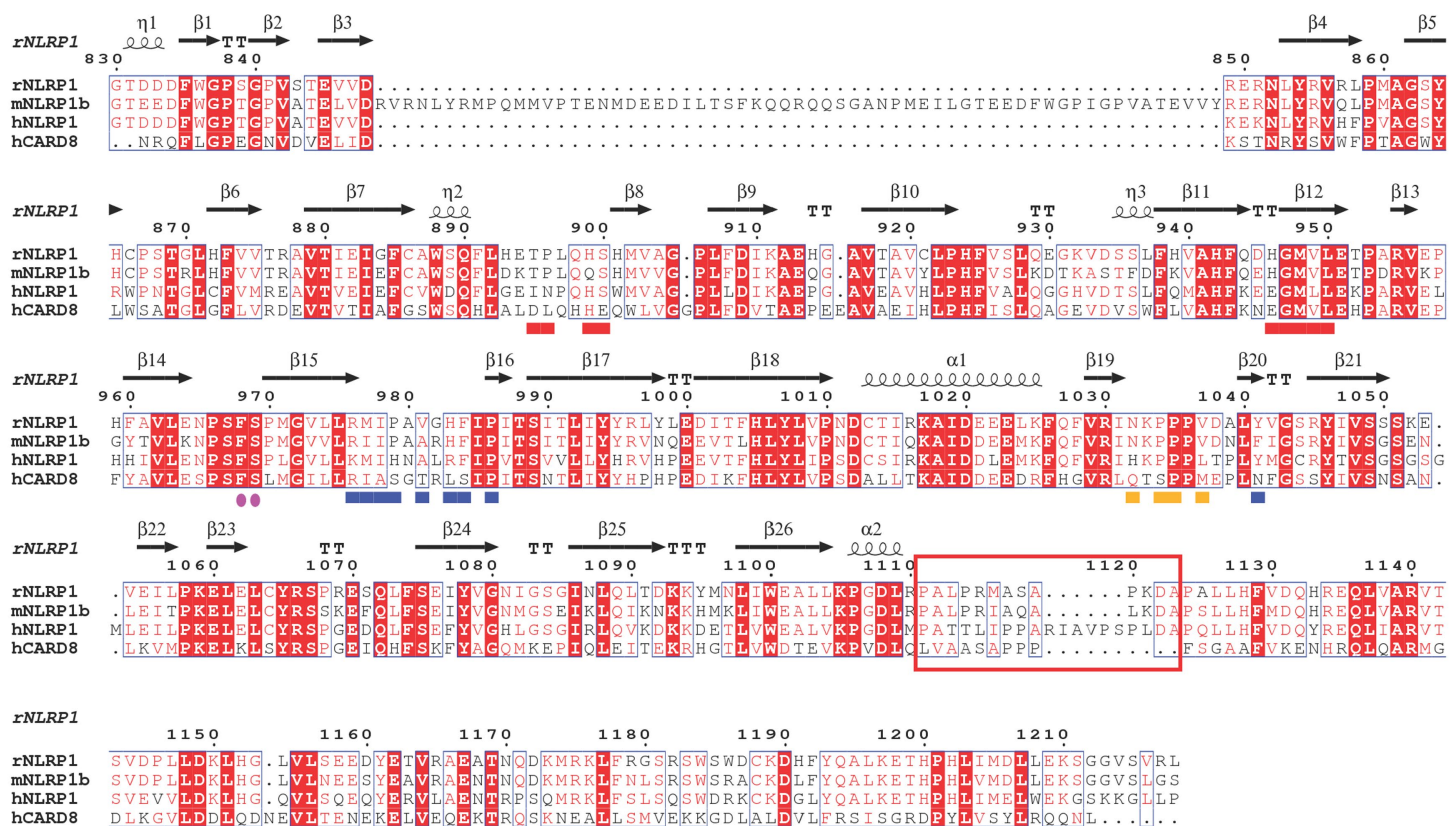

Extended Data Fig. 1 | Complete autocleavage of rNLRP1 FIIND in crystals and sequence alignment among rNLRP1, hNLRP1, NLRP1B and CARD8.

a, Electron density around the active site of rNLRP1 FIIND. The autocleavage site is between F968 and S969 of rNLRP1. b, SDS-PAGE analysis of the crystals of rNLRP1FIIND. The crystals were collected and dissolved in SDS-PAGE buffer. Lanes 1-4 represent crystals from four different wells. c, Structural alignment of rNLRP1 FIIND and UNC5b. The crystal structure of rNLRP1 FIIND was aligned with that of UNC5b (PDB code:3G5B). The colour code is indicated on the figure.d, Sequence alignment of the FIIND-CARD domains of rNLRP1, hNLRP1, NLRP1B and CARD8. The rDPP9-interacting residues around the ZU5-binding site, the UPA-binding site and residues at the UPA homodimeric interface are highlighted with red, blue and yellow squares, respectively. The two residues from the catalytic FS motif are indicated by purple dots, and the linker region between UPA and CARD is highlighted within the red box. See Supplementary Fig. 1 for gel raw data. 


\section{Article}

a

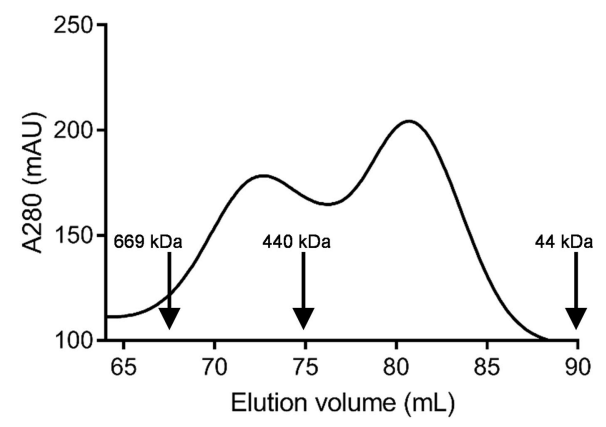

Elution volume $(\mathrm{mL})$

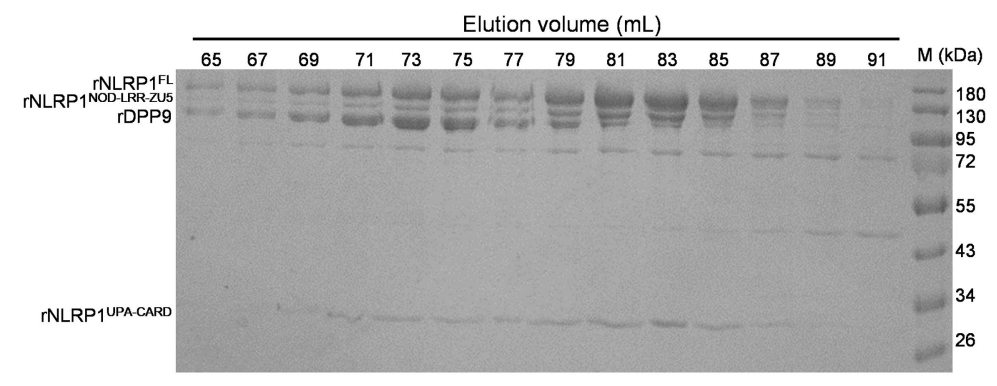

b

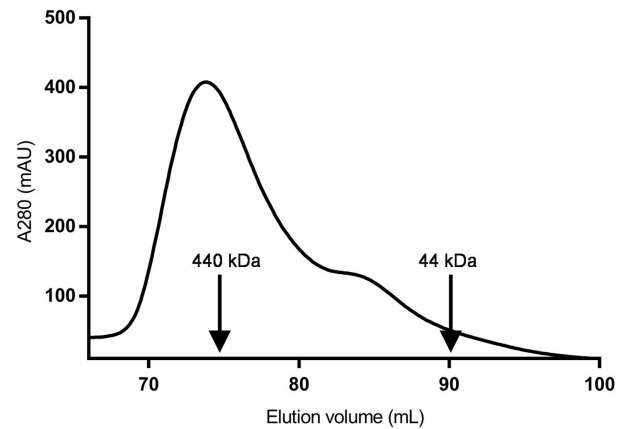

Extended Data Fig. 2 | Gel-filtration analysis of the rNLRP1-rDPP9 and the CARD8-hDPP9 interactions. a, Full-length GST-rNLRP1 and rDPP9 were expressed in insect cells. The complex was purified through GS4B resin. After elution, GST was removed using precision protease and the complex was

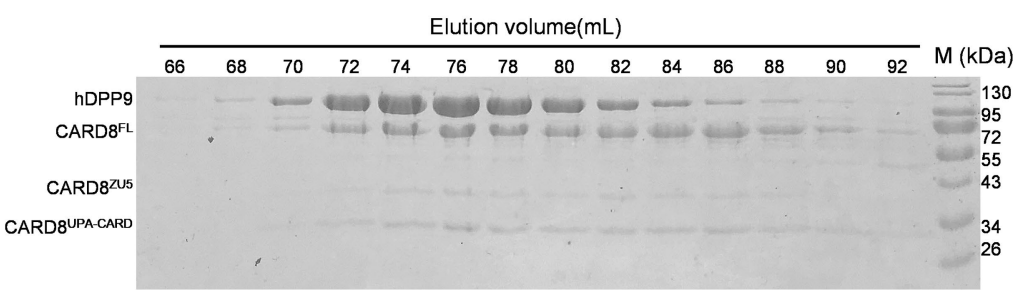

subjected to gel filtration. Left, the gel-filtration profile of the complex. Right, the protein fractions were visualized by SDS-PAGE followed by Coomassie-blue staining. b, The interaction between full-length GST-CARD8 and hDPP9 was assayed as described in a. See Supplementary Fig. 1 for gel raw data. 


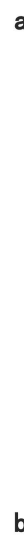

b
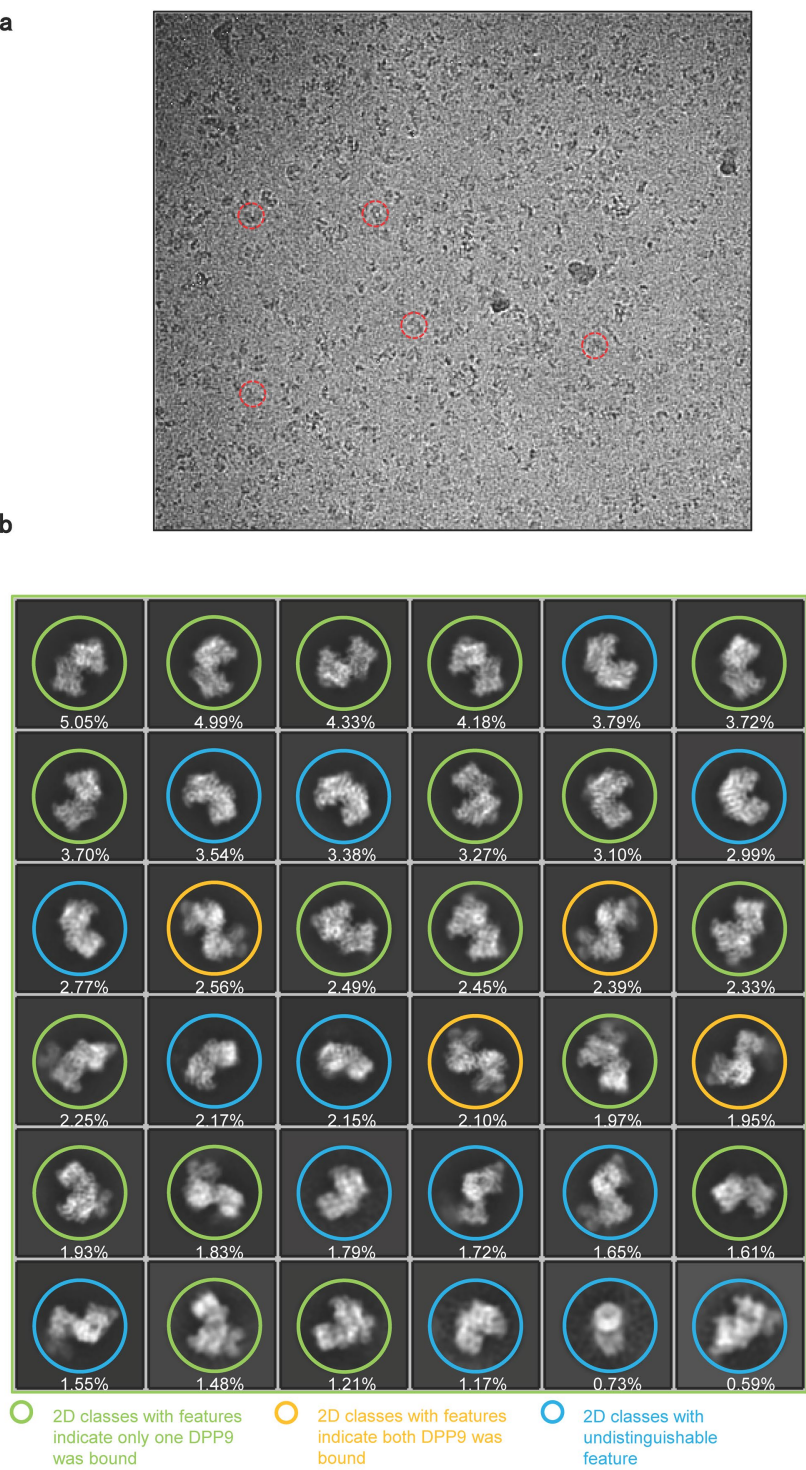

d

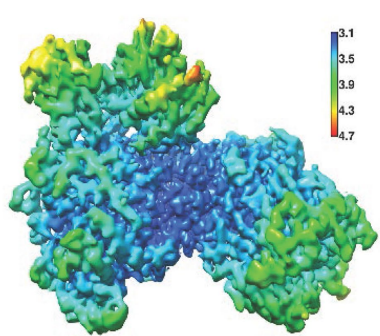

c
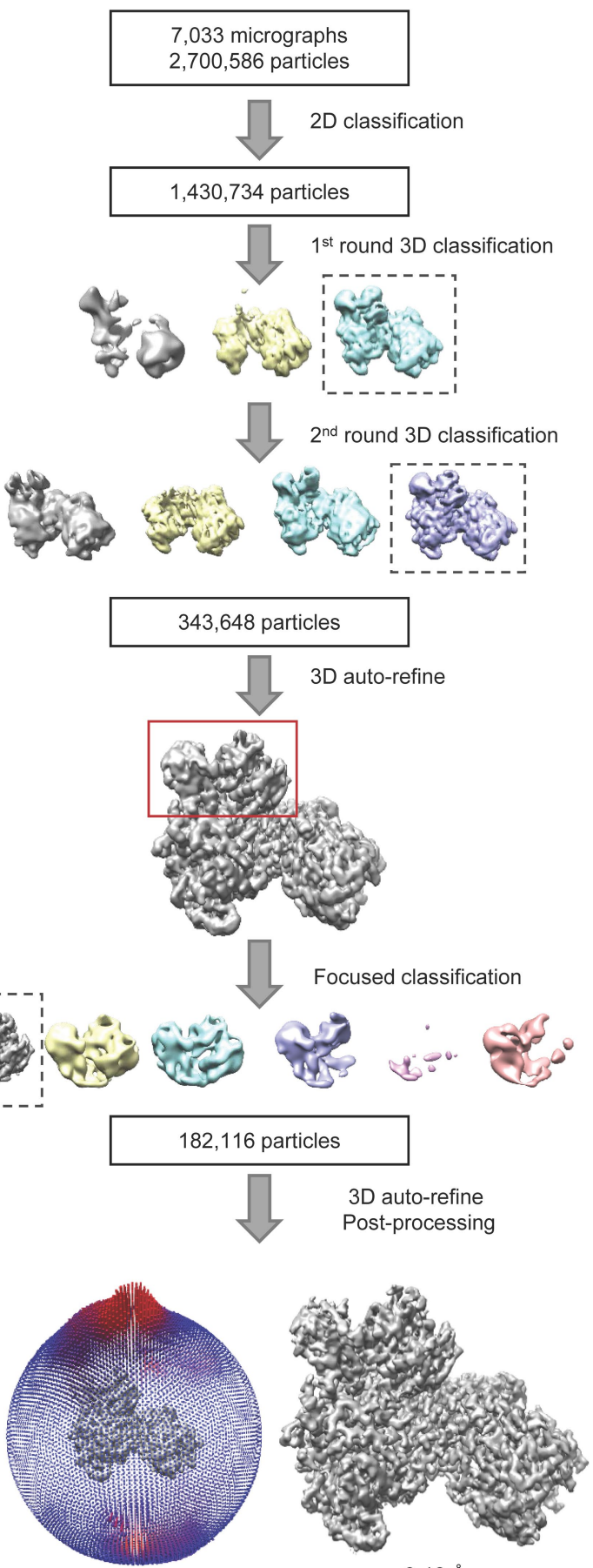

$3.18 \AA$
Extended Data Fig. 3 |3D reconstruction of the rNLRP1-rDPP9 complex.

a, A representative cryo-EM image of the rNLRP1-rDPP9 complex.

b, Representative views of 2D class averages of the rNLRP1-rDPP9 complex used for 3D reconstruction. 2D classes with different rDPP9-binding features are shown in different colours. c, Flow chart of cryo-EM data processing and 3D reconstruction of the rNLRP1-rDPP9 complex.d, The final EM density map of the rNLRP1-rDPP9 complex. The colour code shows the local resolution estimated using Relion. e, Fourier shell correlation curves at 0.143 for the final model of the rNLRP1-rDPP9 complex unmasked (red) or masked (black), and at 0.5 for rNLRP1-rDPP9 global model refined against the 3.18 Å rNLRP1-rDPP9 global map (green), the first half map (blue) and the second half map (cyan). The small difference between the blue and cyan curves after the estimated resolution indicates no overfitting of the coordinate refinement. 


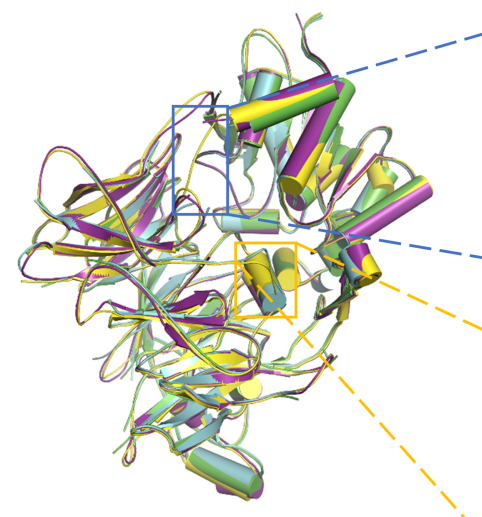

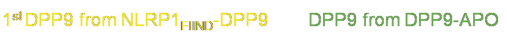

$2^{\text {nd }}$ DPP9 from NLRP1 $1_{\text {FINo }}-$ DPP9

complex

DPP9 from DPP9-1G244 b

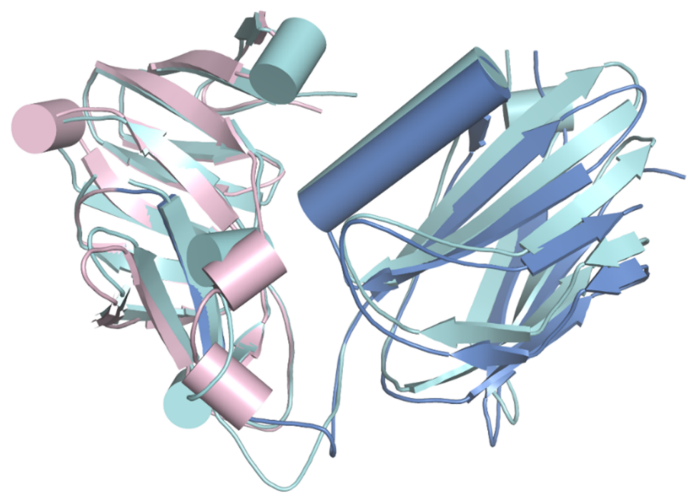

;

c
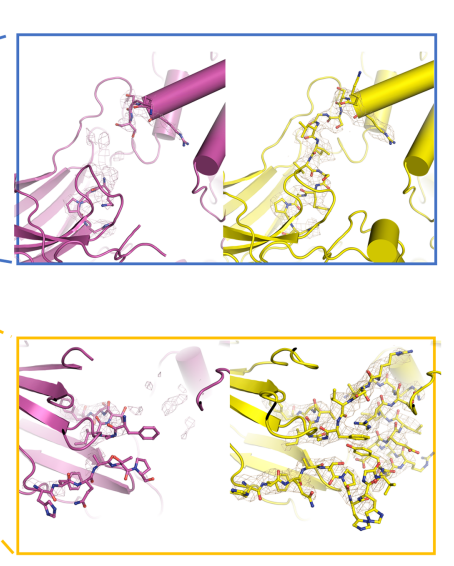

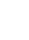
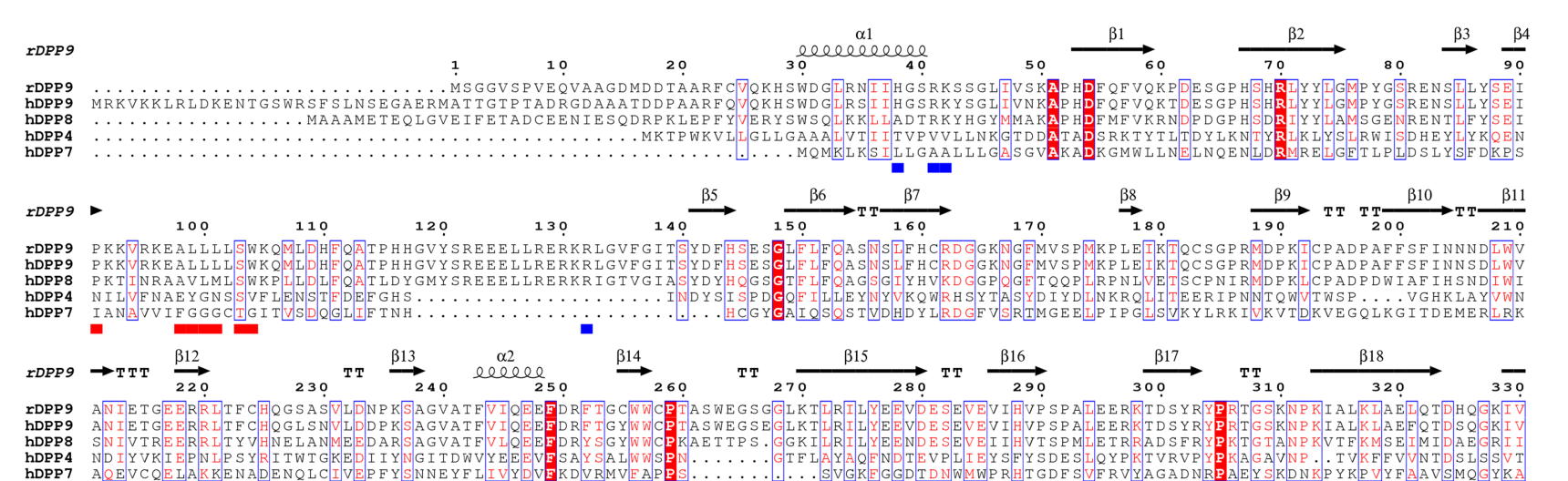

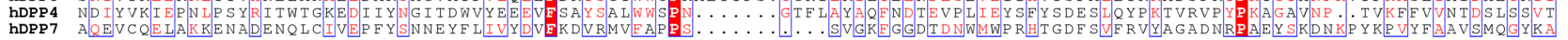

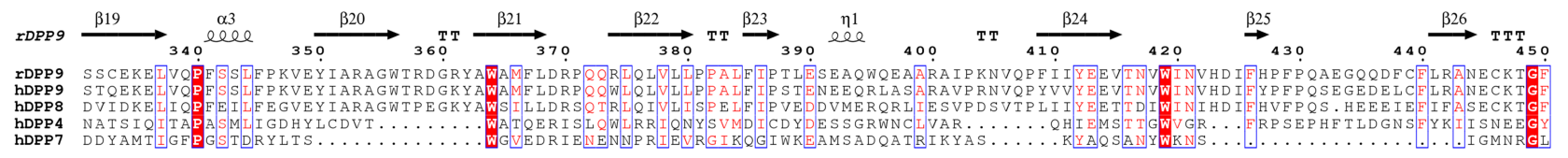

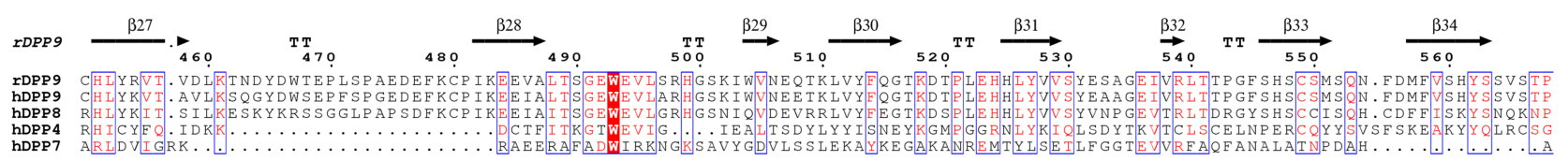

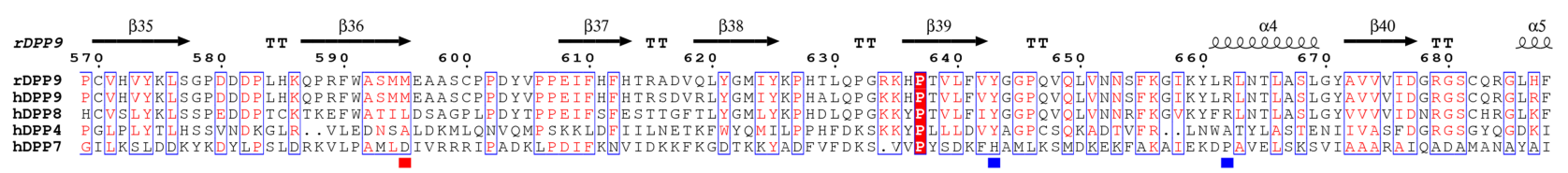

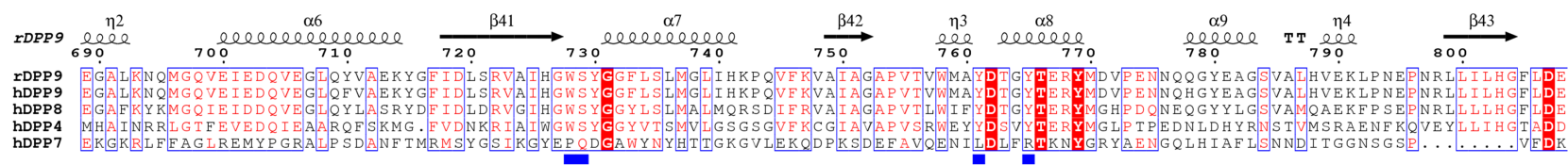

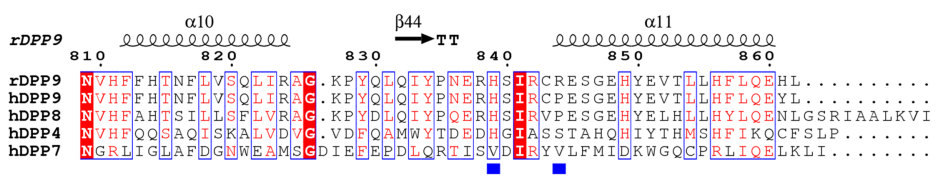

Extended Data Fig. 4 | Structural and sequence alignment. a, Left, structural alignment between the first rDPP9, second rDPP9, apo-hDPP9 and hDPP9$1 \mathrm{G} 244$ (in cartoon representation). The colour code is indicated on the figure. Better-defined loop regions in the rNLRP1-bound rDPP9 subunit are highlighted with open frames. Right, electron density around the stabilized loop regions induced by rNLRP1. All densities are generated in PyMOL and contoured at 5 (region coloured blue) or 7 (region coloured yellow) sigma. b, Alignment of the cryo-EM structure of the rNLRP1 FIIND (with ZU5 shown in pink and UPA in blue) with the crystal structure of the rNLRP1FIIND (in cyan). c, Sequence alignment of rDPP9, hDPP9, hDPP8, hDPP4 and hDPP7. The rNLRP1-interacting residues from the ZU5- and UPA-binding site are marked with red and blue squares, respectively. 
$\mathrm{rNLRP}_{\text {FIIND }}$ monomer

$$
\begin{aligned}
& \text { [Sequence] } \\
& \text { Ser Pro Met Gly Val }
\end{aligned}
$$

[Estimated Sequence]

\begin{tabular}{|lrrrrr|}
\hline & 1 & 2 & 3 & 4 & 5 \\
\hline 1st & Ser & Pro & Met & Gly & Val \\
\hline 2nd & Gly & Asp & Thr & Tyr & Leu \\
4th & Asp & Phe & Ile & Asn & Ala \\
Reliability(\%) & His & Glu & Leu & Glu & Tyr \\
Relu & 20.9 & 100.0 & 79.0 & 72.4 & 100.0 \\
\hline
\end{tabular}

[Evaluated Value]

$\begin{array}{lrrrrr}\text { [Evaluated Value] } & & & & \\ & 1 & 2 & 3 & 4 & 5 \\ \text { Asp } & 5.52 & 3.19 & 0.64 & 0.70 & 1.12 \\ \text { Glu } & 0.73 & 1.08 & 1.04 & 1.12 & 1.11 \\ \text { Asn } & 0.24 & 0.52 & 0.67 & 1.20 & 0.78 \\ \text { Gln } & 3.88 & 0.44 & 1.39 & 0.63 & 0.96 \\ \text { Ser } & 18.30 & 0.11 & 0.18 & 0.54 & 1.00 \\ \text { Thr } & 2.24 & 0.47 & 2.24 & 0.41 & 1.25 \\ \text { His } & 4.59 & 0.23 & 0.92 & 0.00 & 0.00 \\ \text { Gly } & 7.23 & 0.51 & 0.74 & 11.65 & 0.44 \\ \text { Ala } & 2.61 & 0.29 & 1.42 & 0.38 & 1.43 \\ \text { Tyr } & 0.87 & 0.86 & 1.04 & 1.40 & 1.26 \\ \text { Arg } & 1.29 & 0.20 & 1.23 & 0.62 & 0.83 \\ \text { Met } & 1.25 & 0.88 & 19.94 & 0.21 & 0.18 \\ \text { Val } & 0.62 & 0.98 & 1.15 & 0.84 & 41.15 \\ \text { Pro } & 0.03 & 47.23 & 0.32 & 0.27 & 0.47 \\ \text { Trp } & 1.08 & 0.45 & 0.00 & 0.00 & 0.00 \\ \text { Phe } & 0.83 & 1.19 & 1.40 & 0.62 & 0.91 \\ \text { Lys } & 1.05 & 0.73 & 1.37 & 0.18 & 0.39 \\ \text { lle } & 0.95 & 1.06 & 2.15 & 0.83 & 0.94 \\ \text { Leu } & 0.98 & 1.00 & 1.74 & 0.58 & 2.33\end{array}$

rNLRP $_{\text {FIIND }}$-rDPP9 complex

[Sequence]

Ser Pro Met Gly Val

[Estimated Sequence]

\begin{tabular}{|lrrrrr|}
\hline 1st & 1 & 2 & 3 & 4 & 5 \\
\hline 2nd & Ser & Pro & Met & Gly & Val \\
3rd & Leu & Ile & Ile & Asn & Leu \\
4th & Gln & Ala & Tyr & Ile & Ala \\
Reliability(\%) & Gly & Glu & Glu & Glu & Tyr \\
& 36.9 & 68.6 & 81.2 & 48.8 & 100.0 \\
\hline
\end{tabular}

[Evaluated Value]

$\begin{array}{lrrrrr} & 1 & 2 & 3 & 4 & 5 \\ \text { Asp } & 0.20 & 1.93 & 0.58 & 0.78 & 0.84 \\ \text { Glu } & 0.69 & 2.33 & 1.77 & 1.32 & 0.80 \\ \text { Asn } & 0.05 & 0.48 & 0.89 & 2.78 & 0.55 \\ \text { Gin } & 3.18 & 0.50 & 0.88 & 0.78 & 0.86 \\ \text { Ser } & 19.37 & 0.19 & 0.31 & 0.83 & 0.90 \\ \text { Thr } & 1.09 & 0.92 & 1.65 & 1.07 & 0.73 \\ \text { His } & 2.49 & 0.14 & 0.84 & 0.34 & 1.01 \\ \text { Gly } & 2.87 & 0.88 & 0.60 & 13.75 & 0.38 \\ \text { Ala } & 0.64 & 2.49 & 0.73 & 0.77 & 2.10 \\ \text { Tyr } & 0.93 & 1.09 & 2.37 & 1.03 & 1.87 \\ \text { Arg } & 0.42 & 2.09 & 0.88 & 0.69 & 0.73 \\ \text { Met } & 1.01 & 0.99 & 25.91 & 0.31 & 0.02 \\ \text { Val } & 0.86 & 1.22 & 1.41 & 1.26 & 37.73 \\ \text { Pro } & 0.06 & 26.14 & 0.40 & 0.45 & 1.33 \\ \text { Trp } & 1.82 & 0.60 & 0.38 & 0.00 & 0.00 \\ \text { Phe } & 0.57 & 1.84 & 0.68 & 1.27 & 1.00 \\ \text { Lys } & 1.14 & 0.17 & 1.18 & 0.25 & 1.09 \\ \text { Ile } & 0.43 & 3.37 & 3.41 & 2.13 & 0.58 \\ \text { Leu } & 4.71 & 0.40 & 1.00 & 1.13 & 2.40\end{array}$

b

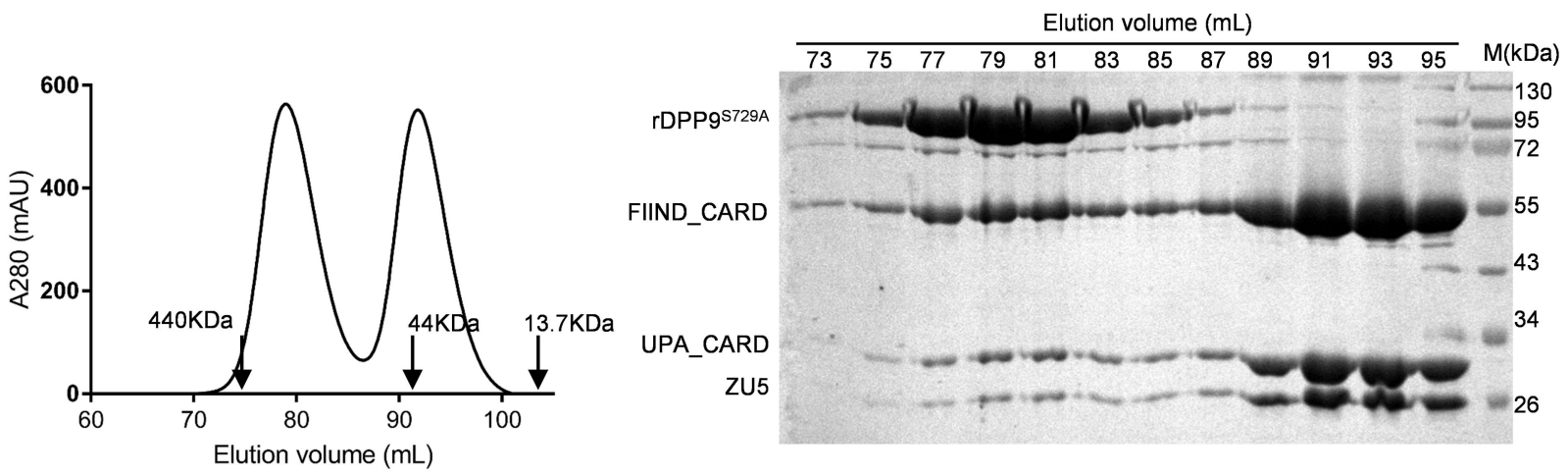

c
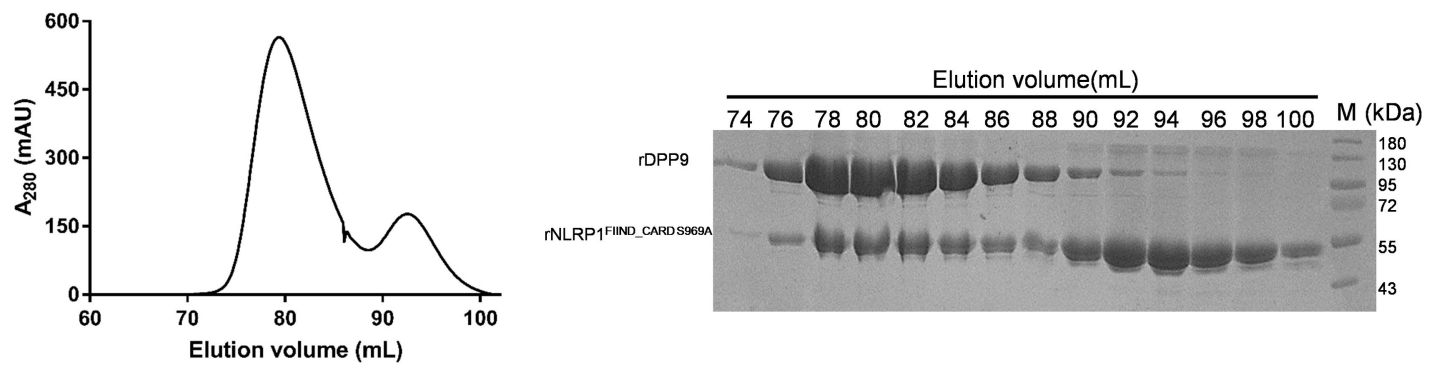

Extended Data Fig. 5 | See next page for caption. 


\section{Article}

Extended Data Fig. 5 | Functional assays to analyse the influence of DPP9 protease activity on the interaction between DPP9 and NLRP1.

a, N-terminal sequence analysis of rNLRP1 FIIND by Edman degradation. Shown on the left and the right are N-terminal sequences of UPA from rNLRP1FIIND and rNLRP1 FIIND-rDPP9, respectively. b, Catalytic activity of rDPP9 is dispensable for the binding of rDPP9 to rNLRP1. The catalytic mutant rDPP9(S729A) with GST fused at the N terminus was co-expressed with rNLRP1 FIIND-CARD in insect cells. The complex was purified through GS4B resin. After elution, GST was removed using precision protease and the complex was subjected to gel filtration. Left, the gel-filtration profile of the complex. Right, the protein fractions were visualized by SDS-PAGE followed by Coomassie-blue staining. c, Gel-filtration analysis of the autoprocessing-deficient S969A mutant of rNLRP1FIIND-CARD in complex with rDPP9. The two proteins were co-expressed in insect cells and purified using affinity chromatography. The purified complex was further cleaned using gel filtration. Left, the gel-

filtration profile of the complex. Right, the fractions of the two proteins were visualized by SDS-PAGE followed by Coomassie-blue staining. See Supplementary Fig. 1 for gel raw data. 
a

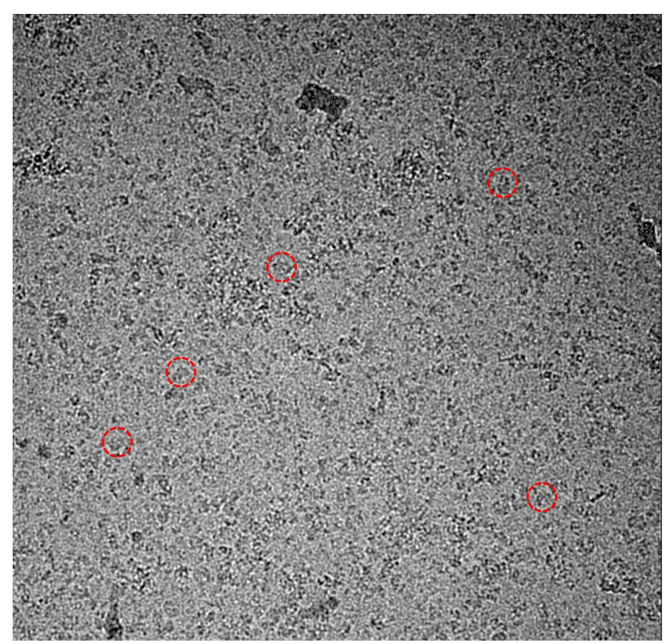

d

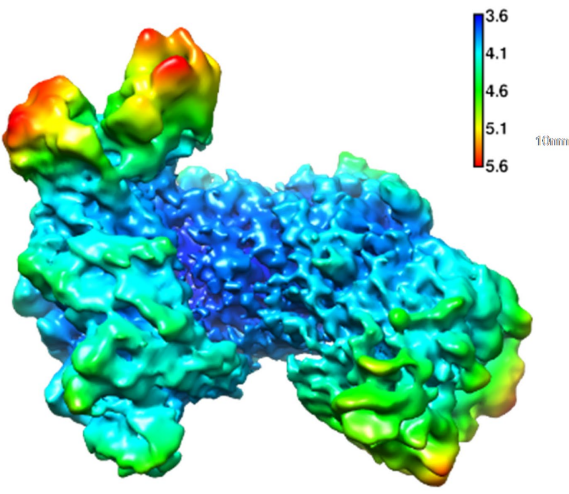

e

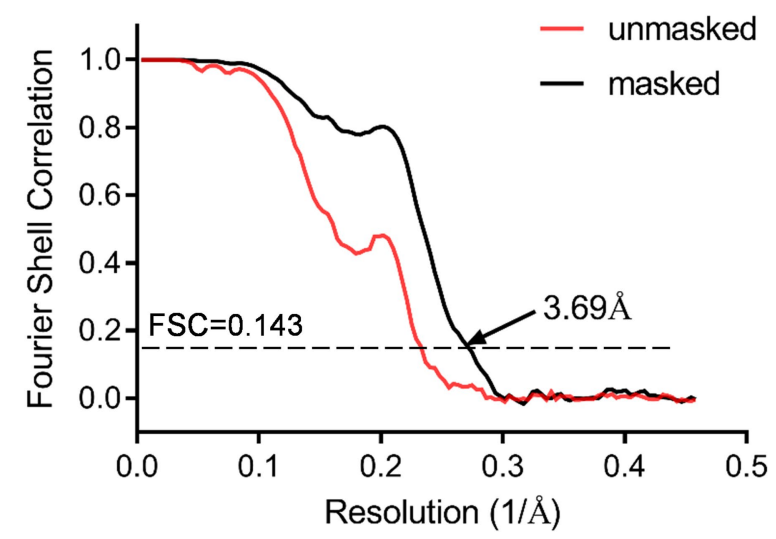

Extended Data Fig. 6 |3D reconstruction of the rNLRP1

FIIND-CARD(S969A)-rDPP9 complex. a, A representative cryo-EM image of the rNLRP1 FIIND-CARD(S969A)-rDPP9 complex.b, Representative 2D class averages of the rNLRP1FIIND-CARD(S969A)-rDPP9 complex.c, Flow chart of cryo-EM data processing and 3D reconstruction of the rNLRP1FIIND- b

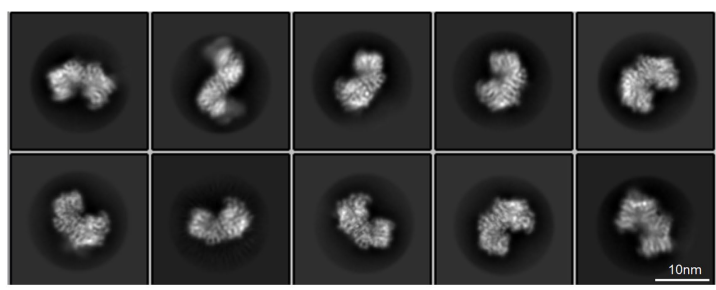

c

4,677 micrographs

$1,725,380$ particles

2D classification

\section{$1,117,656$ particles}
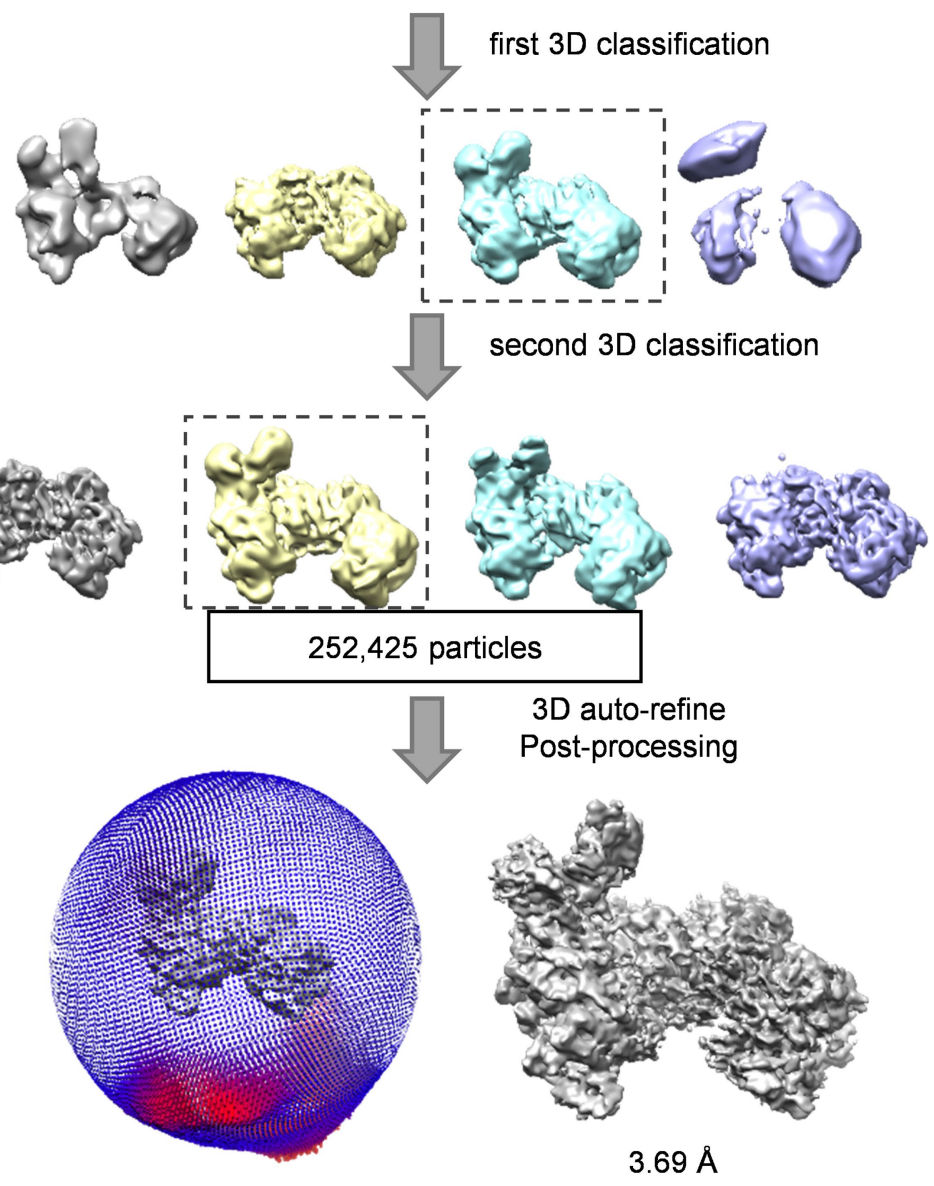

CARD(S969A)-rDPP9 complex. d, The final EM density map of the rNLRP1 FIIND-CARD(S969A)-rDPP9 complex. The colour code shows the local resolution estimated using Relion.e, Fourier shell correlation curve (at 0.143) of the final reconstruction of the rNLRP1FIIND-CARD(S969A)-rDPP9 complex. 


\section{Article}

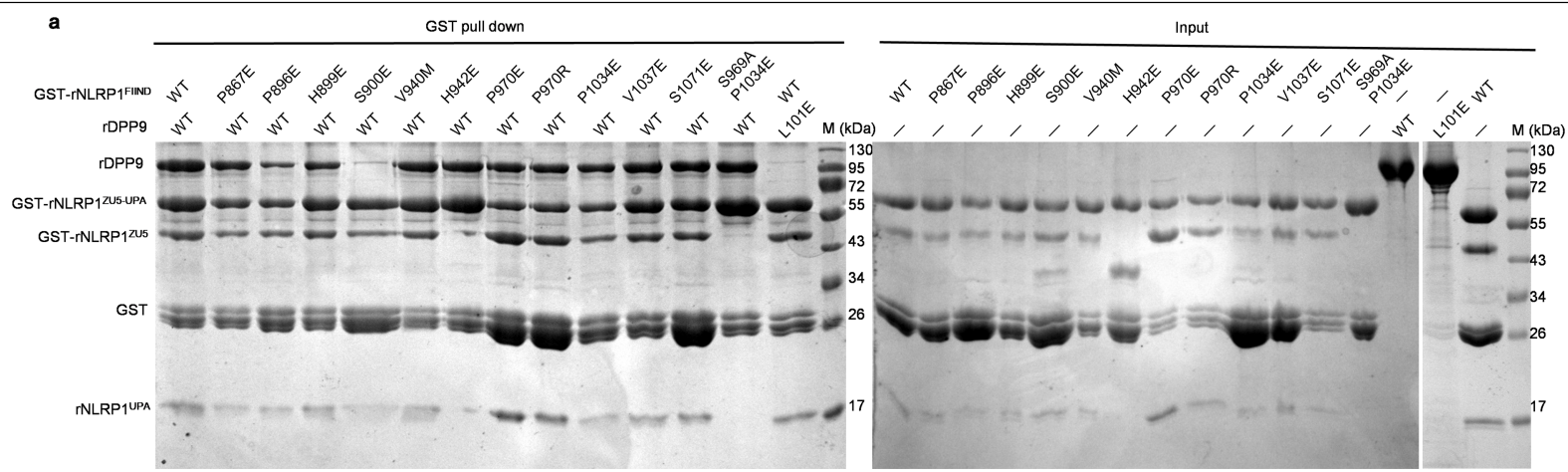

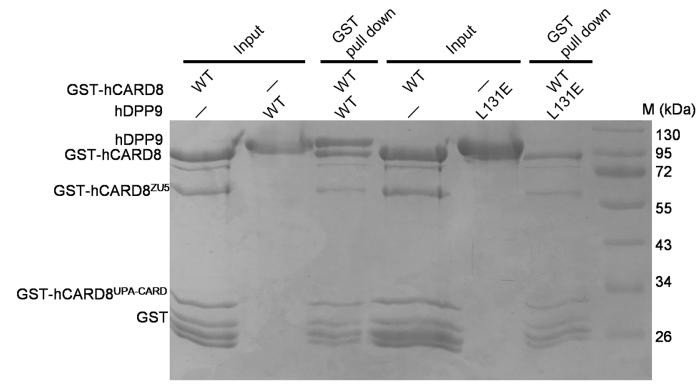

d

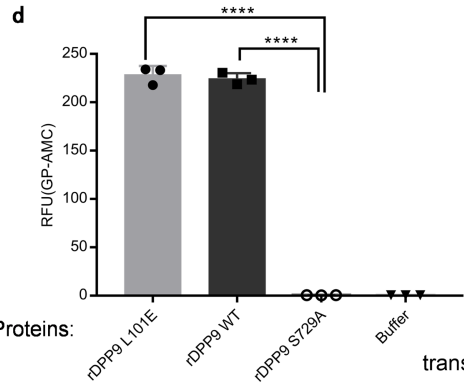

DPP8/9 dKO 293T-ASC-GFP

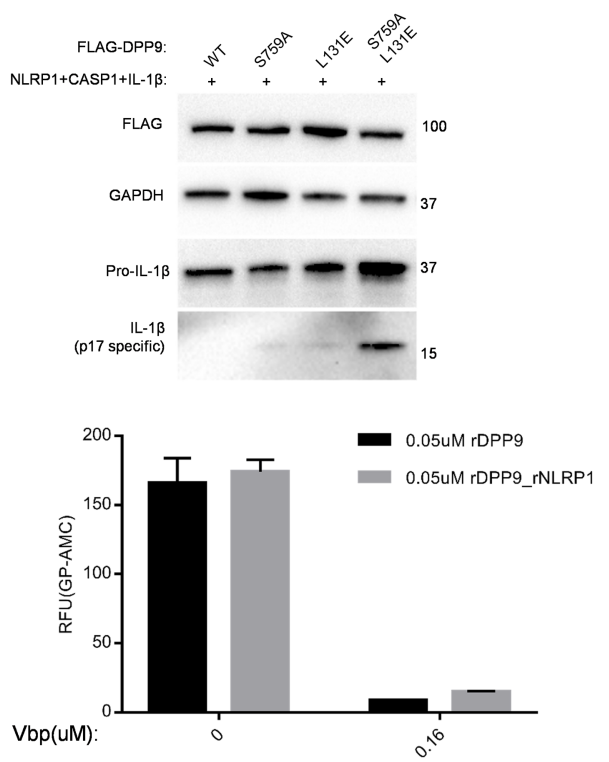

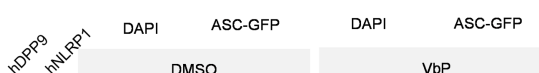

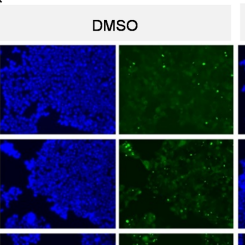

VbP

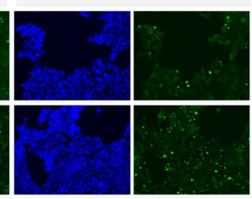

S759A
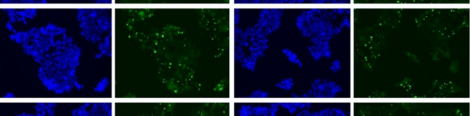

L131E
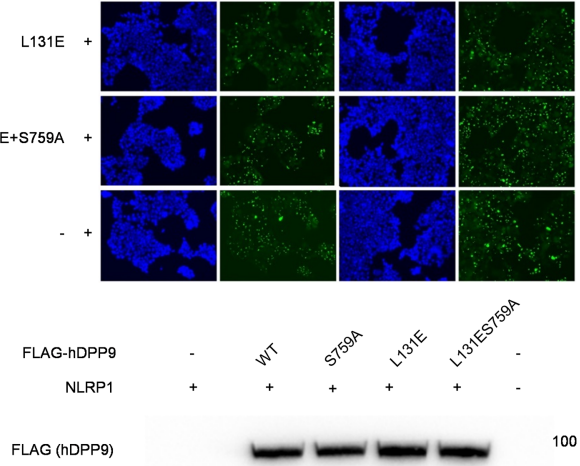

FLAG (hDPP9)

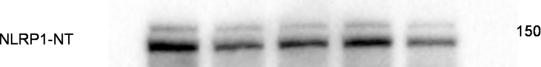

GAPDH

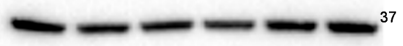

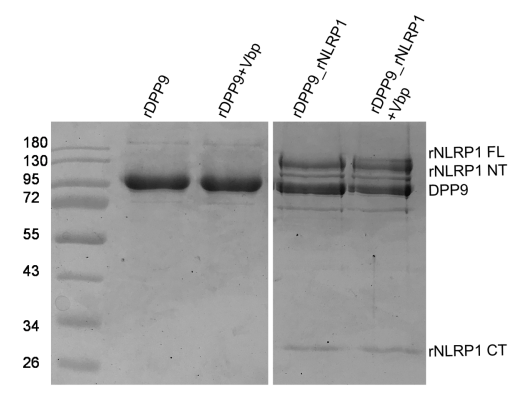

Extended Data Fig. 7|See next page for caption. 
Extended Data Fig. 7 | DPP9 interacts with FIIND from rNLRP1 and CARD8. a, Mutagenesis analysis of the ZU5-binding site of rDPP9. N-terminally GSTfused wild-type or mutant rNLRP1 FIIND and rDPP9 were individually purified from insect cells. Wild-type or mutant rNLRP1FIIND was used to pull down rDPP9 (including rDPP9(L101E)) with GS4B resin. After extensive washing, proteins bound to the GS4B resin were visualized by SDS-PAGE followed by Coomassie-blue staining. b, The interaction of hDPP9 with CARD8.

$\mathrm{N}$-terminally GST-fused CARD8 was used to pull down non-tagged wild-type or mutant hDPP9 in vitro. c, Top, representative images of DPP8/DPP9 doubleknockout ASC-GFP 293T cells transfected with hNLRP1 and wild-type hDPP9 or hDPP9 mutants. Bottom, expression of wild-type and DPP9 mutants in DPP8/
DPP9 double-knockout 293T cells. The soluble lysate was blotted for DPP9, NLRP1 or GAPDH.d, Mutation of L101 of rDPP9 (left) or its equivalent L131 of hDPP9 (right) has no effect on the protease activity. Each experiment was repeated at least three times. One-way ANOVA, ${ }^{* * * *} P<0.0001$. RFU, relative fluorescence units. e, DPP8/DPP9 double-knockout 293T cells were cotransfected with $3 \times$ Flag hDPP9 variant together with CASP1-Myc and pro-IL-1 $\beta$. ASC-GFP speck formation was monitored for consistency with that in c. Lysates were analysed by immunoblotting.f, Left, inhibition of the protease activity of rDPP9 and rNLRP1-rDPP9 by VbP. Right, SDS-PAGE analyses of the rDPP9 and rNLRP1-rDPP9 complexes used in the activity assays. See Supplementary Figs. 2 and 4 for gel raw data. 
a

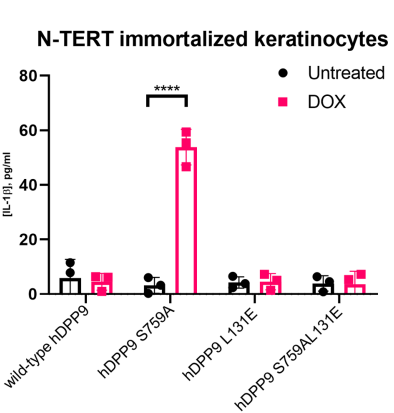

b

293T-ASC-GFP-hNLRP1 (hDPP8/9 +/+

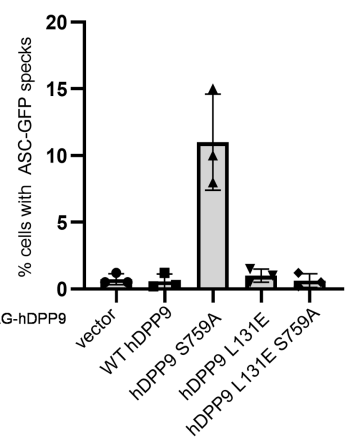

Vector

DAPI ASC-GFP

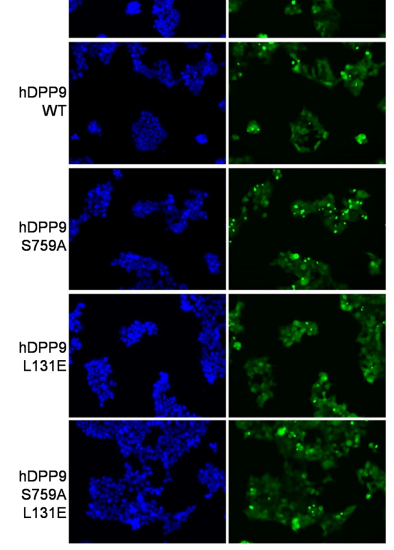

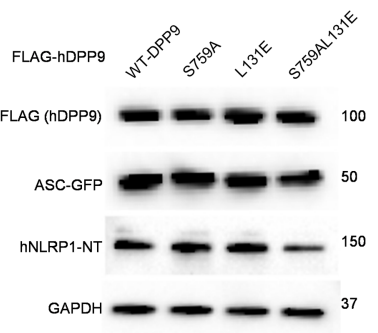

c
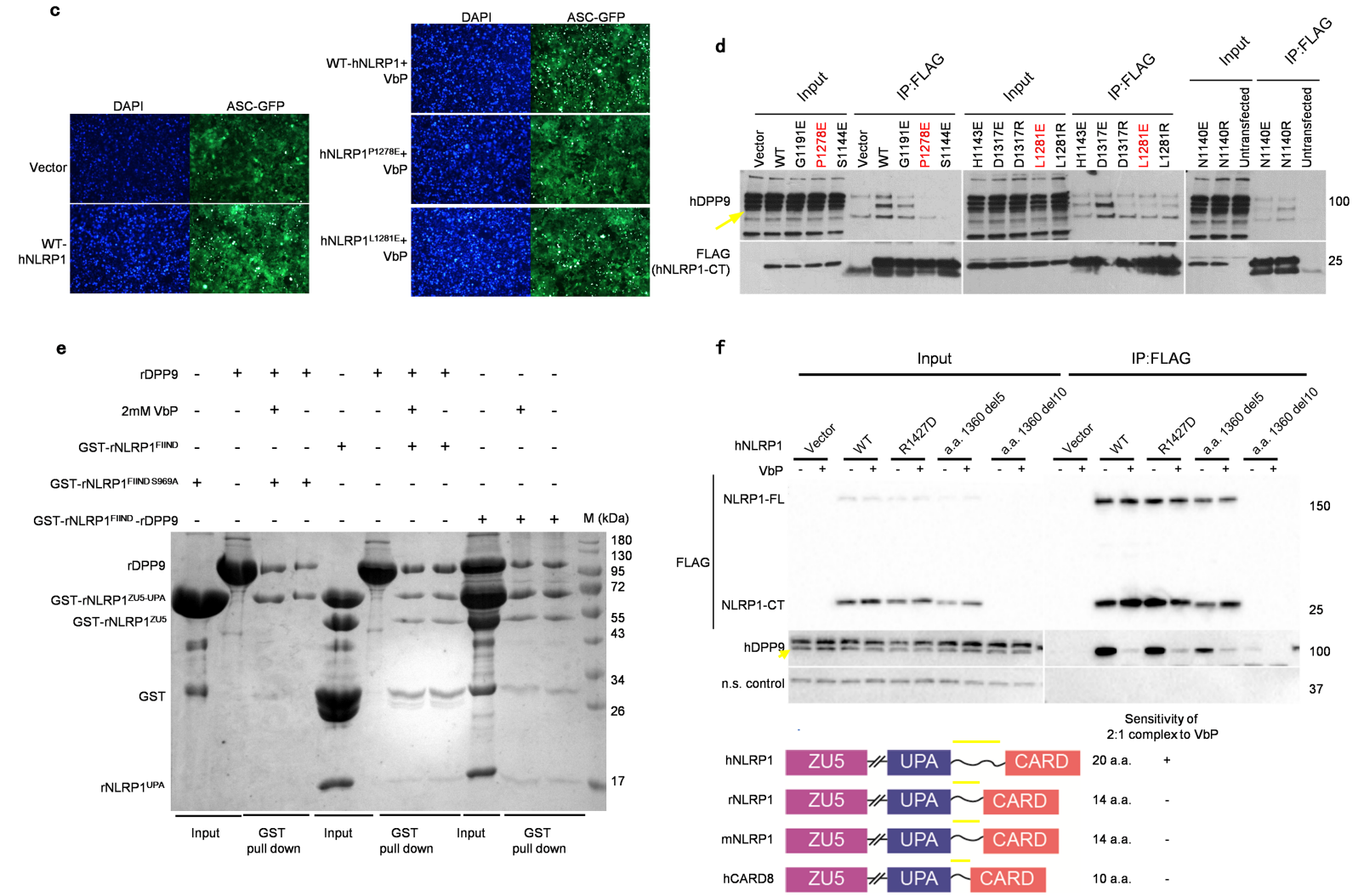

g
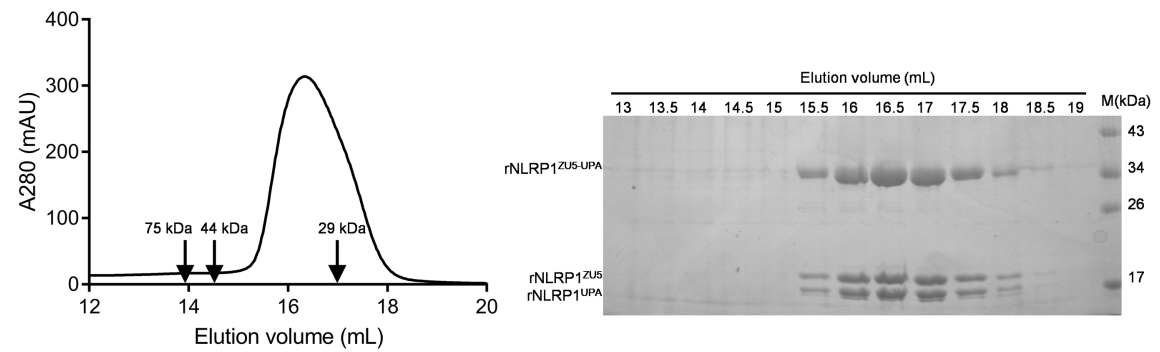

Extended Data Fig. 8 |See next page for caption. 
Extended Data Fig. 8 | Additional evidence for the supression of NLRP1 by DPP9 binding and enzymatic activity. a, Immortalized keratinocytes stably transduced with Tet-ON-3×Flag hDPP9 lentiviruses were treated with or without doxycycline for $24 \mathrm{~h}$. Conditioned media were subjected to anti-IL-1 $\beta$ ELISA. The $y$ axis represents the concentrations of IL-1 $\beta$ in the medium. Bar graphs represent data from three inductions. Two-way ANOVA, ${ }^{* * *} P<0.0001$. b, Middle, representative images of 293T cells expressing ASC-GFP and hNLRP1 and transfected with wild-type hDPP9 or hDPP9 mutants. Left, the percentage of cells with ASC-GFP specks was quantified using more than 200 cells (left). Right, the expression of wild-type hDPP9 or hDPP9 mutants in 293T cells expressing ASC-GFP and NLRP1. The soluble lysate was blotted for hDPP9, hNLRP1-NT, ASC-GFP or GAPDH. Each experiment was repeated at least three times. c, Representative images of ASC-GFP expressing 293T cells transfected with the indicated hNLRP1 mutants. $\operatorname{VbP}(3 \mu \mathrm{M})$ was added $24 \mathrm{~h}$ post transfection. Cells were fixed $48 \mathrm{~h}$ post transfection and the nuclei were counterstained with Hoescht 33342. The percentage of cells with ASC-GFP specks was quantified using more than 200 cells. d, ASC-GFP293T cells transfected with C-terminally Flag-tagged hNLRP1 constructs. Anti-Flag immunoprecipitation was performed on approximately $1 \mathrm{mg}$ whole-cell lysate $48 \mathrm{~h}$ after transfection. e, GST-fused rNLRP1 FIIND and rDPP9 were individually purified from insect cells. Wild-type or mutant rNLRP1 FIIND proteins were used to pull down rDPP9 with GS4B resin in the presence or in the absence of VbP.f, Top, 293T cells were transfected with the indicated hNLRP1-Flag mutants. Anti-Flag immunoprecipitation was performed as in d. The hDPP9 band is indicated with the yellow arrow. The linker region between hNLRP1UPA and CARD begins at amino acid 1360. Bottom, comparison of the linker regions of hNLRP1, rNLRP1, NLRP1B and hCARD8. The linker length and the sensitivity of the respective 2:1 complex to $\mathrm{VbP}$ are shown on the right. $\mathrm{g}$, The gel filtration profile (left) and SDS-PAGE (right) of the rNLRP1 FIIND. See Supplementary Figs. 2 and 5 for gel raw data. 


\section{Article}

Extended Data Table 1 | Crystallography data collection and refinement statistics

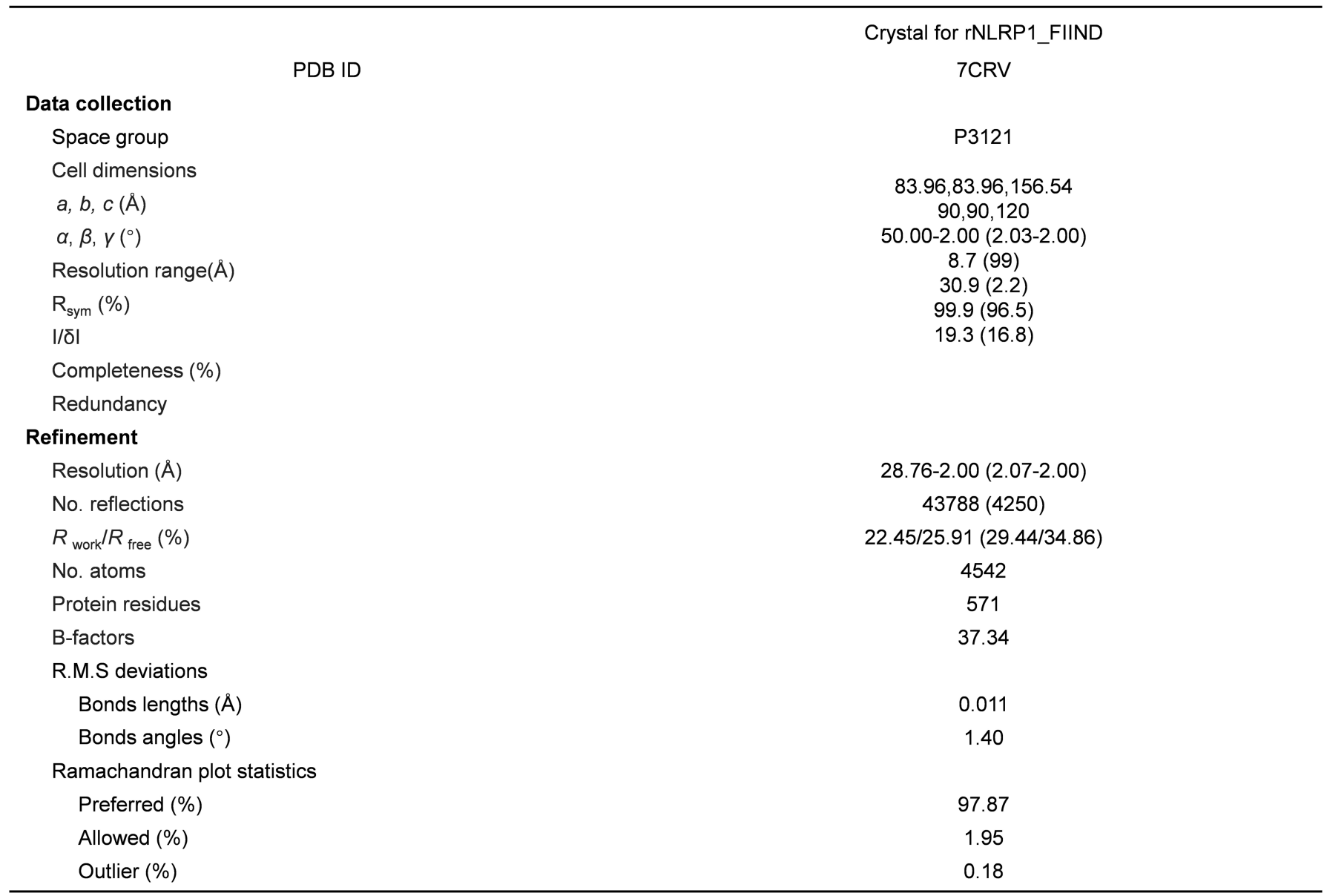


Extended Data Table 2 | Cryo-EM data collection, 3D reconstruction and model statistics

\begin{tabular}{|c|c|c|}
\hline & rNLRP1-rDPP9 & rNLRP1 FIIND-CARD(S969A)-rDPP9 \\
\hline PDB ID & 7CRW & NA \\
\hline EMDB ID & EMD-30458 & EMD-30459 \\
\hline \multicolumn{3}{|l|}{ Data collection } \\
\hline Cryo electron microscope & FEI Titan Krios & FEI Titan Krios \\
\hline Pixel size $(\AA)$ & 1.061 & 1.091 \\
\hline Total electron dose $\left(\mathrm{e}^{-} / \AA^{2}\right)$ & 50 & 50 \\
\hline Exposure rate (e-/pixel/sec) & 10 & 11 \\
\hline \multicolumn{3}{|l|}{ 3D reconstruction } \\
\hline Software & RELION 3.1 & RELION 3.1 \\
\hline Total extraced particles & $2,700,586$ & $1,725,380$ \\
\hline Number of particles used for 3D reconstruction & 182,116 & 252,425 \\
\hline Symmetry imposed & $\mathrm{C} 1$ & $\mathrm{C} 1$ \\
\hline Resolution range $(\AA)$ & $3.06-4.65$ & $3.60-6.14$ \\
\hline Resolution $(\AA)$ after refinement & $3.64(\mathrm{FSC}=0.143)$ & $4.29(\mathrm{FSC}=0.143)$ \\
\hline Resolution $(\AA)$ after post-processing & $3.18(\mathrm{FSC}=0.143)$ & $3.69(\mathrm{FSC}=0.143)$ \\
\hline Map sharpening B-factor $\left(\AA^{2}\right)$ & -50 & -100 \\
\hline \multicolumn{3}{|l|}{ Model composition } \\
\hline Rwork/Rfree (\%) & $33.41 / 33.41$ & NA \\
\hline B factors & 80.08 & NA \\
\hline \multicolumn{3}{|l|}{ R.M.S deviations } \\
\hline Bonds lengths $(\AA)$ & 0.005 & NA \\
\hline Bonds angles $\left({ }^{\circ}\right)$ & 0.723 & NA \\
\hline MolProbity score & 1.90 & NA \\
\hline Clash score & 9.23 & NA \\
\hline Rotamer outliers (\%) & 0.60 & NA \\
\hline EMRinger score & 3.00 & NA \\
\hline \multicolumn{3}{|l|}{ Ramachandran plot statistics } \\
\hline Preferred (\%) & 93.91 & NA \\
\hline Allowed $(\%)$ & 5.94 & NA \\
\hline Outlier (\%) & 0.15 & NA \\
\hline
\end{tabular}




\section{Reporting Summary}

Nature Research wishes to improve the reproducibility of the work that we publish. This form provides structure for consistency and transparency in reporting. For further information on Nature Research policies, see our Editorial Policies and the Editorial Policy Checklist.

\section{Statistics}

For all statistical analyses, confirm that the following items are present in the figure legend, table legend, main text, or Methods section.

n/a Confirmed

$\square$ The exact sample size $(n)$ for each experimental group/condition, given as a discrete number and unit of measurement

$\square$ \ A statement on whether measurements were taken from distinct samples or whether the same sample was measured repeatedly

The statistical test(s) used AND whether they are one- or two-sided

$\square$ Only common tests should be described solely by name; describe more complex techniques in the Methods section.

Х $\square$ A description of all covariates tested

$\square$ \A description of any assumptions or corrections, such as tests of normality and adjustment for multiple comparisons

$\square$ A full description of the statistical parameters including central tendency (e.g. means) or other basic estimates (e.g. regression coefficient)

$\bigotimes$ AND variation (e.g. standard deviation) or associated estimates of uncertainty (e.g. confidence intervals)

$\varnothing$ For null hypothesis testing, the test statistic (e.g. $F, t, r$ ) with confidence intervals, effect sizes, degrees of freedom and $P$ value noted

$\triangle$ Give $P$ values as exact values whenever suitable.

Х $\square$ For Bayesian analysis, information on the choice of priors and Markov chain Monte Carlo settings

Х $\square$ For hierarchical and complex designs, identification of the appropriate level for tests and full reporting of outcomes

X $\square$ Estimates of effect sizes (e.g. Cohen's $d$, Pearson's $r$ ), indicating how they were calculated

Our web collection on statistics for biologists contains articles on many of the points above.

\section{Software and code}

Policy information about availability of computer code

Data collection Blu-Ice BL19U1, AutoEmation.

Data analysis RELION 3.1, MotionCor2 1.0.5, CTFFIND 4.1.12, CCP4i 7.1, Phenix 1.18, Chimera 1.14, Coot 0.8.9, Pymol 4.6.0, HKL2000 v718, Image J1.8.0

For manuscripts utilizing custom algorithms or software that are central to the research but not yet described in published literature, software must be made available to editors and reviewers. We strongly encourage code deposition in a community repository (e.g. GitHub). See the Nature Research guidelines for submitting code \& software for further information.

\section{Data}

Policy information about availability of data

All manuscripts must include a data availability statement. This statement should provide the following information, where applicable:

- Accession codes, unique identifiers, or web links for publicly available datasets

- A list of figures that have associated raw data

- A description of any restrictions on data availability

All relevant data are available from the authors and/pr included in the manuscript of Supplementary Information. Atomic coordinates and the EM map have been deposited in the Protein Data Bank (PDB) and Electron Microscopy Data Bank (EMDB) under accession number 7CRW, 7CRV, EMD-30458 and EMD-30459. 


\section{Field-specific reporting}

Please select the one below that is the best fit for your research. If you are not sure, read the appropriate sections before making your selection. \Life sciences $\square$ Behavioural \& social sciences Ecological, evolutionary \& environmental sciences

For a reference copy of the document with all sections, see nature.com/documents/nr-reporting-summary-flat.pdf

\section{Life sciences study design}

All studies must disclose on these points even when the disclosure is negative.

Sample size For ASC speck assays, at least three transfections were carried out. A minimum of 100 cells were scored for the presence of ASC-GFP specks.

Data exclusions Data exclusion for particles in bad 2D or 3D classes with no defined features was a standard processing for EM data.

Replication To ensure reproducibility of experimental findings, each assay was performed at least three times to confirm the results.

Randomization Animals or human research participants were not involved in this study and, as such, samples were not randomized for the experiments.

Blinding Animals or human research participants were not involved in this study and, as such, samples were not blinded for the experiments.

\section{Reporting for specific materials, systems and methods}

We require information from authors about some types of materials, experimental systems and methods used in many studies. Here, indicate whether each material, system or method listed is relevant to your study. If you are not sure if a list item applies to your research, read the appropriate section before selecting a response.

Materials \& experimental systems

Methods

n/a Involved in the study

$\square$ Antibodies

\Eukaryotic cell lines

\ $\square$ Palaeontology and archaeology

$\mathrm{n} / \mathrm{a}$ Involved in the study

$\searrow \square$ ChIP-seq

$\bigotimes \square$ Flow cytometry

$\bigotimes \square$ MRI-based neuroimaging

Х $\square$ Animals and other organisms

\ $\square$ Human research participants

\ $\square$ Clinical data

$\bigotimes \square$ Dual use research of concern

\section{Antibodies}

Antibodies used

c-Myc Antibody (9E10): sc-40, Santa Cruz Biotechnology; FLAG antibody (M2), F3165, Sigma-Aldrich; Human NLRP1 antibody (AF6788), R\&D systems;anti-IL-1B p17 specific (CST, \#83186S);GAPDH (Santa Cruz Biotechnology, \#sc-47724);IL-1ß (R\&D systems, \#AF-201); anti-FLAG tag (SigmaAldrich, F3165).DPP9 antibody - Catalytic domain (Abcam, ab42080) ASC antibody (Adipogen, AL177)

AffiniPure Goat Anti-Mouse IgG (H+L) (Code: 115-035-166,Jackson Immunoresearch)

Peroxidase AffiniPure Goat Anti-Rabbit IgG (H+L) (Code: 111-035-144,Jackson Immunoresearch)

All primary antibodies were used at $300 \mathrm{ng} / \mathrm{mL}$. All secondary antibodies were used at $1 / 7,500(\mathrm{v} / \mathrm{v})$.

Validation

All epitope tag and ASC antibodies had been validated by the manufacturers. NLRP1-NT antibody was validated using NLRP1overexpressing 293T cells. DPP9 antibody had been previously validated using DPP8/9 knockout cells in Zhong et al, JBC, 2018.

\section{Eukaryotic cell lines}

Policy information about cell lines

Cell line source(s)

Sf21 cells lines from Thermo Fischer Scientific. https://www.thermofisher.com/order/catalog/product/12682019?SID=srchsrp-12682019\#/12682019?SID=srch-srp-12682019.

293T ( ATCC CRL-3216).

Immortalised human keratinocytes (N/TERT-1 or N-TERT herein) were provided by H. Rheinwald (MTA to Skin Research Institute of Singapore).

Authentication

Sf21 cell lines were verified by manufacturer's website and Identity of these cell lines were frequently checked by their morphological features. https://www.thermofisher.com/order/catalog/product/12682019?SID=srch- 
srp-12682019\#/12682019?SID=srch-srp-12682019.

293T was validated by STR test (ATCC).

$\mathrm{N}$-TERT immortalized keratinocytes were not authenticated.

Mycoplasma contamination

Commonly misidentified lines (See ICLAC register)
Sf21 cell line was not tested for mycoplasma contamination.

Human cells are routinely tested for mycoplasma contamination (average once every two months).

All human cell lines were tested for mycoplasma contaminated every two months using MycoGuard ${ }^{\text {TM }}$ Mycoplasma PCR Detection Kit (Genecopoeia)

No commonly misidentified cell lines are used in this study. 